\title{
Performance Optimization of Integrated Resilience Engineering and Lean Production Principles
}

\author{
Ali Azadeh \\ Professor of Industrial Engineering, School of Industrial Engineering, University of Tehran, Tehran, \\ Iran \\ Email Address: aazadeh@ut.ac.ir \\ Reza Yazdanparast
}

Ph.D. Student of Industrial Engineering, School of Industrial Engineering, University of Tehran,

Tehran, Iran

Email Address: r.yazdanparast@ut.ac.ir

Saeed Abdolhossein Zadeh

MSc of Industrial Engineering, School of Industrial Engineering, University of Tehran, Tehran, Iran

Email Address: saeed.prsl@gmail.com

\begin{abstract}
Afshin Esmail Zadeh
MSc of Industrial Engineering, School of Industrial Engineering, University of Tehran, Tehran, Iran Email Address: a.esmaei1388@yahoo.com
\end{abstract}

\begin{abstract}
This paper conducts performance assessment from integrated resilience engineering (IRE) and lean production points of view. This is the first study that evaluates the impact of integrated resilience engineering (IRE) on lean production principles. Second, this study considers integrated impact of lean production by a unique intelligent algorithm. The proposed algorithm is composed of radial basis function (RBF), multi-layer perceptron (MLP) and adaptive neuro-fuzzy inference system (ANFIS). Moreover, the algorithm is capable of handling both crisp and fuzzy data due to the existence of intelligent approach. The proposed algorithm is equipped with verification and validation mechanism through conventional regression, statistical methods and data envelopment analysis. To demonstrate the applicability of the study, a real-world pipe manufacturer is considered as our case study. The results showed that "pull system" and "fault tolerant” among lean and IRE factors,
\end{abstract}


respectively have been implemented inappropriately, while other factors are either suitably executed or ineffective.

Keywords: Integrated resilience engineering (IRE); Lean production; Artificial neural networks (ANNs); Adaptive neuro-fuzzy inference system (ANFIS); Data envelopment analysis (DEA)

\section{Introduction}

Resilience engineering (RE) and lean production are two critical concepts that have rarely been discussed concurrently, particularly in the production context. This is surprising given that they have a similar motivation for improvement of processes and service delivery. There has certainly been a growing interest in both $\mathrm{RE}$ and lean production in recent yearsdue to the focus on the efficiency of production systems. In this paper, we investigate how they could work together in order to achieve mutual benefits.

\subsection{Definition and key assumptions of lean}

Lean manufacturing/ production derived mostly from the Toyota Production System (TPS). It has been defined in many ways according to the suitability of the research context. However, it is still based on the original goal of TPS which is "to do more with less". In the definition of lean, three concepts of Muda, Muri and Mura have been introduced. Muda is waste on the scope of lean or non-value adding activities. Muri refers to a poor work condition and Mura represents unevenness in demand.

The philosophy of this theory is to remove non-value adding steps (wastes). The initial manufacturing wastes were defined by Ōno (1988) as follows:

- Transport: Moving products that are not actually required to perform the processing;

- Inventory: All components, works-in-process, and finished products not being processed;

- Motion: People or equipment moving or walking more than is required to perform the processing;

- Waiting: Waiting for the next production step, interruptions of production during shift change;

- Overproduction: Production ahead of demand;

- Over Processing: Resulting from poor tool or product design creating activity;

- Defects: Effort involved in inspecting for and fixing defects. 
It is possible to define lean through five 'lean principles' as follows (Womack \& Jones, 2010):

- Customer orientation: Determining what customer exactly expects and wants;

- Reduce waste: Investigating each product value stream and then determining all nonvalue added steps;

- Standard product: Standardizing all the processes after designing the most efficient product flow;

- Pull system: This principle is used when continuous flow is impossible in a product flow. It is employed by considering product demand and by enabling backwards in product value chain;

- Task management: This factor seeks to remove non-value adding steps and use resources such as time and information efficiently.

Lean concept is well accepted for improving efficiency and eliminating wastes in production systems (Fliedner, 2008). Over the last decade there has been a quick and sudden increase in the use of lean (Brandao de Souza, 2009). It is important to know that lean principles are not designed just for waste reduction. Besides reducing wastes, and consequently costs, lean seeks to improve the efficiency of production system by increasing value-adding activities in product flow (Govindan, Azevedo, Carvalho, \& Cruz-Machado, 2015; Hines, Holweg, \& Rich, 2004). Ng, Vail, Thomas, and Schmidt (2010) used lean principles in order to improve the quality of care in an emergency department without any additional resources. Hicks, McGovern, Prior, and Smith (2015) applied lean principles to the design of healthcare facilities and demonstrated the applicability and efficiency of the lean principles. Lunardini et al. (2014) optimized instruments' utilization in a spine surgery medical center by employing lean principles. Aqlan and Ali (2014) integrated lean principles, failure mode and effects analysis (FMEA), and fuzzy analysis for improving risk assessment process in a chemical plant. FMEA is one of the first highly structured, systematic techniques for failure analysis. Min (2014) investigated the impact of lean implementation on performance of healthcare systems. Susilawati, Tan, Bell, and Sarwar (2015) performed lean assessment in manufacturing systems in Indonesia. They identified 66 effective parameters in lean assessment, and used standard questionnaire and fuzzy based method for measuring the leanness of manufactories. Sharma, Dixit, and Qadri (2015) investigated the impact of lean production on performance of machine tool companies by determining lean criteria and using 
factor analysis and multiple regression analysis. At the end, they determined criteria with significant positive and negative impact on companies’ performance.

Although lean principles have served as an improvement tool for manufacturing and service systems, many researchers have shown that companies' tendencies to find low-cost solutions may have led them to leaner but more vulnerable environment (Azevedo et al., 2008; Peck, 2005). Besides, turbulence and volatility are main characters of today's market and manufacturing systems. Therefore, manufacturing systems need to be resilient in order to survive (Carvalho et al., 2011). Therefore, considering resilience engineering (RE) along with lean production principles is required for high volume and low cost productions.

\subsection{Resilience Engineering}

Resilience engineering (RE) is a new concept which seeks safety and performance improvement in organizations (Wreathall, 2006). It helps organizations to make their system much more reliable and sustainable in case of any disturbance. According to Nemeth et al. (2008), a resilient organization can predict, deal with, recover and learn from unanticipated threats. Therefore, the advantages of RE is twofold: (1) it precludes failures, losses and damages, and (2) it is capable of reacting efficiently soon after these phenomena took place (Nemeth, Wears, Woods, Hollnagel, \& Cook, 2008).

Resilient organizations function proactively. It means that they don't only have high levels of immunity but also are able to react to any changes which deflect system's performance from the planned one (Haimes, 2009)(Hollnagel, Braithwaite, \& Wears, 2013). The classical factors of RE are as follows: (Hollnagel, Woods, \& Leveson, 2007; Woods \& Hollnagel, 2006; Wreathall, 2006).

- Top management commitment: This principle indicates management's willingness to invest, assign resources and a devotion to improve RE above or to the same degree as the organization's other goals.

- Reporting culture: Without a just culture of reporting, the willingness of the staff to report problems and subjects will be much decreased. Hence, the ability of the association is limited to learn about weaknesses in its late defenses.

- Learning: System should be able to learn from past events in order to respond effectively. It is a culture which should be implemented in system's structure.

- Awareness: Management should allow the personnel to participate in system's information regarding failures, so they can be aware of what happened, why happened 
and what the consequences are. Keeping this kind of information in a black box and prevent systems’ components to determine new vulnerabilities.

- Preparedness: The association or system actively predicts the problems of human activity in human-machine systems and prepares to deal with them.

- Flexibility: Systems' components should be allowed to deal with failures using their natural instincts, abilities and experiences. This confidence help the personnel to improve their abilities in case of any new failure and disturbance happened, so they can deal with it better. Without flexibility, it is not possible to prepare the system to deal with any new disturbance or failure (Clegg, 2000).

Azadeh, Salehi, Ashjari, and Saberi (2014) incorporated four additional principles into classic RE and called it integrated RE (IRE) as follows:

- Self-organization: In self-organization systems, arrangements come from the performances of interdependent agents who change data, take actions, and non-stop adjust to react about others' activities more than from the imposition of an overall plan by a main authority (Plowman et al., 2007).

- Teamwork: It is one of the most important facilitators in obtaining positive, costeffective results in different organizational and adaptability and productivity. When the workload of system is high, this feature can mitigate the pressure on individuals and organizations(Rasmussen, Pejtersen, \& Goodstein, 1994).

- Redundancy: Based on this factor, there should be a replacement plan for each critical resource such as staff or equipment in case of failure, breakdown or absence (Frangopol \& Curley, 1987).

- Fault-tolerant: This factor is one of the most promising ways of increasing system immunity and reliability. It helps the system to preserve the specified function in the presence of mistakes (Ling-Ling \& Yong-Duan, 2010).

In the past decade, several studies have focused on concept and applicability of RE in different industries. Shirali, Motamedzade, Mohammadfam, Ebrahimipour, and Moghimbeigi (2015) assessed RE factors in a process industry. They employed some other concepts such as risk management to determine poor indicators for process improvement. Hegde et al. (2014)analyzed RE in a healthcaresystem by employing the critical incident interviewing method. Ross et al. (2014) presented a study on a healthcare system by considering resilience and quality of care, they demonstrated that possible failures can cause delay in patient treatment. Jeffcott, Ibrahim, and Cameron (2009) studied RE concept in health care systems 
and proved its effectiveness in safety improvement. Dinh, Pasman, Gao, and Mannan (2012) identified six effective principles in employing $\mathrm{RE}$ and resilience of a process by studying related literature and experts’ judgment. Hansson, Herrera, Kongsvik, and Solberg (2009) demonstrated the application of RE for occupational hazards and injuries reduction, and safety improvement. Azadeh, Salehi, Ashjari, et al. (2014) assessed performance of IRE factors in a petrochemical plant using standard questionnaire and data envelopment analysis approach. Azadeh, Salehi, Arvan, and Dolatkhah (2014) evaluated the performance of RE factors in a petrochemical plant. They evaluated the performance of RE factors in safety improvement in high risk environments.

This paper aims to conduct performance assessment from IRE and lean points of view by an intelligent algorithm based on artificial neural networks (ANNs). To the best of our knowledge, this is the first study that conducts a comprehensive performance assessment from the viewpoints of RE and lean production in a real-world case.

The plan for the remainder of the paper is as follows. Section 2 presents a description of the algorithm used. Implementation of the algorithm on a real-world case study is explained in Section 3. Section 4 provides the results obtained together with some fruitful analyses on them. Lastly, Section 5 is dedicated to concluding remarks and directions for future research.

\section{The proposed algorithm}

Performance assessment of production and service-oriented systems has been a very important issue in past decades. Many efficiency frontier analysis approaches have been presented, however, the restrictive assumptions have been made. Most of the presented approaches have considered deterministic frontier,which is sensitive to outliers. Azadeh et al. (2007) presented a non-parametric efficiency frontier analysis approach based on neural networks. The presented algorithm is been used as a base approach for performance assessment of various industrial systems. Estimating efficiency scores using neural networks empower the approach to deal with stochastic frontier, which makes the results more reliable. The previous algorithm by Azadeh et al. (2007) has been modified with new features in order to provide better performance evaluations. In this paper, an adaptive intelligent algorithm proposed by Azadeh, Ghaderi, Anvari, and Saberi (2007) is employed to assess performance from RE and lean points of view. The algorithm is based on ANNs and statistical methods. In this study,three well-known ANN models namely multilayer perceptron (MLP), radial basis function (RBF), and adaptive neuro-fuzzy inference system (ANFIS) are considered.Data envelopment analysis is also employed for verification and validation of obtained results, 
while perturbation tests are used for determining the optimal DEA model. The main contribution of this study is the presented conceptual model which considers combined resilience engineering factors and lean principles to improve both performance and sustainability. Also, a real world case study is evaluated and the effectiveness of the proposed algorithm is shown. The proposed package for evaluating both RE and lean philosophy is applicable and easy to use and can help managers to improve the resiliency and performance similar manufacturing systems.The flowchart of the presented algorithm in this study for evaluating integrated resilience engineering (IRE) and lean production principles is demonstrated in Figure 1. 


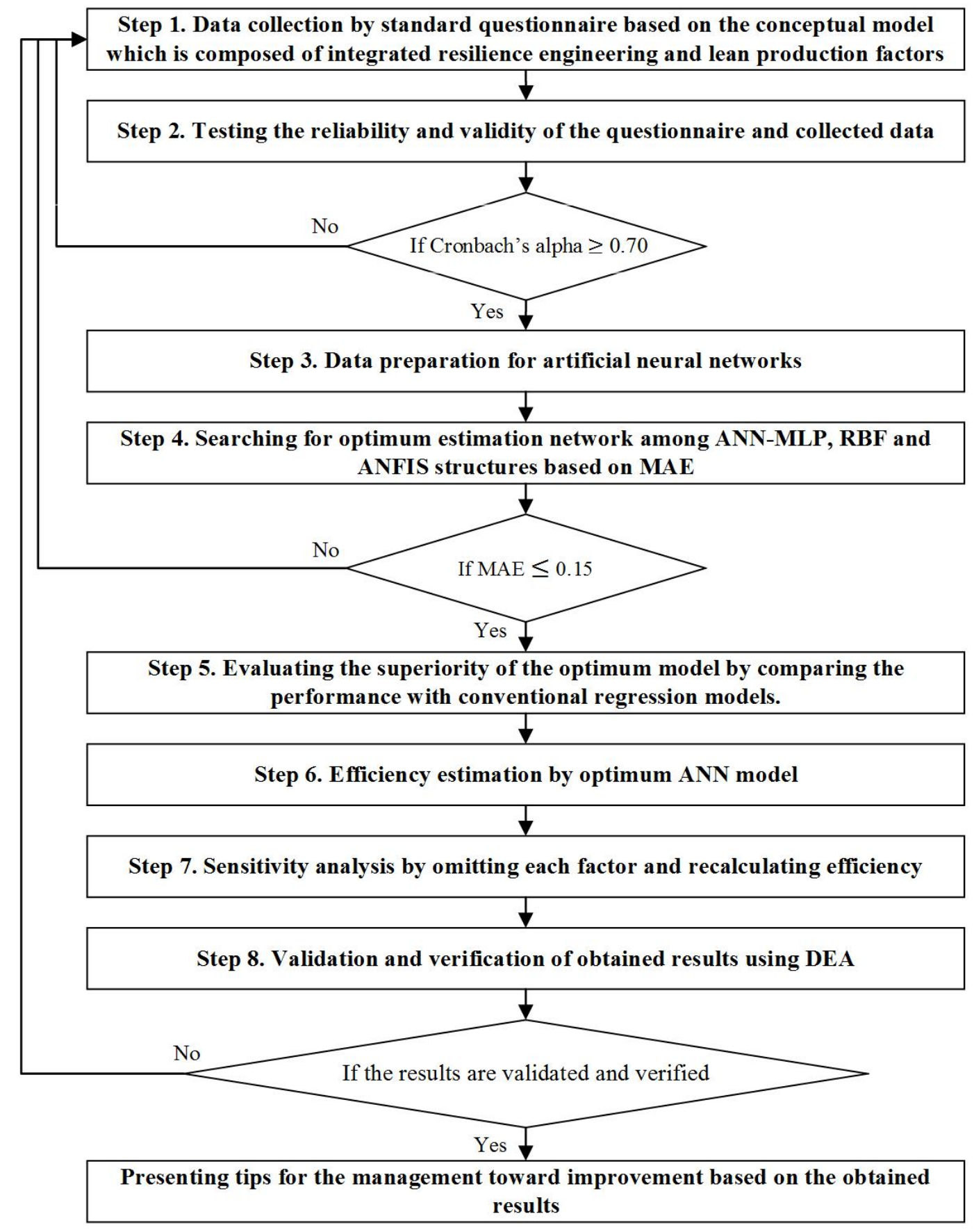

Figure 1.The flowchart of the proposed algorithm in this study

\subsection{Implementation of the algorithm}

The steps of the presented intelligent algorithm along with the nomenclatureused in this paper are as follows:

\section{Indices}

$i \quad$ The index of considered input variables $(i=1,2, \ldots, m)$

$r \quad$ The index of considered output variables $(r=1,2, \ldots, s)$ 
The index of operators $(j=1,2, \ldots, n)$

The index of the DMU with the maximum frontier function shift value

Parameters

$x_{i j}$

The observed value for input variable $i$ and operator $j$

$y_{r j}$

The observed value for output variable $r$ and operator $j$

Variables

$Z_{r j} \quad$ The normalized value of output variable $r$ for operator $j$

$Z_{i j} \quad$ The normalized value of input variable $i$ for operator $j$

$T_{r j}$

The estimated value for output variable $r$ and DMU $j$ using optimum ANN model

$E_{r j}$

The error between the observed output value and estimated output value using ANN for each output variable $r$ and operator $j$

$E_{r j}^{\prime} \quad$ The frontier function shift for output $\mathrm{r}$ and operator $\mathrm{j}$

$E_{v} \quad$ The maximum value for frontier function shift among all DMUs

$T_{r v}$

The estimated value for DMU v (the DMU with the maximum value of frontier function shift)

$S h_{r j} \quad$ The shift value for output variable $r$ and operator $j$

$F_{r j} \quad$ The efficiency score of output variable $\mathrm{r}$ and operator $\mathrm{j}$

$\lambda_{j} \quad$ The degree of benchmarking for $j$ th DMU

$\theta \quad$ The obtained efficiency score using DEA

Step 1.Data Collection:For collecting the required data, standard questionnaires are designed and distributed amongoperators of the system being studied. In order to employ an integrated evaluation and optimization scheme, a conceptual model based on IRE factors and lean production principles is presented. In the presented model,IRE factors are considered as inputs of the model and lean production principles are considered as outputs. The conceptual model of the presented study is explained as follows:

In efficiency frontier analysis, efficiency score is obtained by maximizing outputs while minimizing inputs. Although the concept of considered factors indicates that both IRE and lean factors should be considered as output variables, it appears (mathematically) that IRE factors as inputs provide better efficiency scores.Therefore, Equations 1 and 2 are presented to arrangeinputs (IRE) and outputs (lean production) correctly.Since, input variables should be smaller the better (STB) type while outputs should be larger the better (LTB) type. The 
following equations are presented torange the values between 0 to 1 (normalization), and fix their types simultaneously (Bashiri, Farshbaf-Geranmayeh, \& Mogouie, 2013). Equation (2) is applicable for lean production principles, while Equation (1) changes the type of the data along with its scale. Therefore, IRE factors are changed into STB type using Equation (1).

$$
\begin{aligned}
& Z_{i j}=\frac{\operatorname{Max}\left(x_{i j}\right)-x_{r j}}{\operatorname{Max}\left(x_{r j}\right)-\operatorname{Min}\left(x_{r j}\right)} j=1,2, \ldots, n ; i=1,2, \ldots, m \\
& Z_{r j}=\frac{y_{r j}-\operatorname{Min}\left(y_{r j}\right)}{\operatorname{Max}\left(y_{r j}\right)-\operatorname{Min}\left(y_{r j}\right)} j=1,2, \ldots, n ; r=1,2, \ldots, s
\end{aligned}
$$

Where $Z_{r j}$ is the normalized value of the output variable $r$ for operator (DMU) $j$, while $Z_{i j}$ is the normalized value of the input variable $i$ for operator (DMU) $j$. The observed value for output variable $i$ and operator $j$ is indicated by $x_{i j}$, while $y_{r j}$ represents the observed value for output variable $r$ and operator $j$.

Step 2. Reliability and validity: Determine the reliability and validity of the questionnaire by Cronbach’s Alpha and factor analysis, respectively.

Step 3. Data preparation: In this study IRE factors are considered as inputs and lean principles are outputs of the model. Since ANN is used in this study, after gathering required data regarding inputs and outputs of the model, we have to determine a set of data for training and testing of ANN. In this study 30\% (15\% each) of gathered data is considered for test and validation of the model.

Step 4. Determining the best ANN structure for function estimation: In order to find the best structure for estimating outputs of the model and calculating efficiencies, three different artificial neural networks are used. The considered ANN models are compared to each other base on their related mean absolute error (MAE). Different structures have been considered for each model. At the end, the best estimation function is determined. It should be mentioned that the number of training data is changed from 70 to 90 percent of the data set to prevent overtraining (overfitting). Also, each architecture is run 100 times with random data sets to handle possible noise and prevent overfitting. Thus, the obtained MAE value for each model would be the average of MAEs for all the runs with 10 to 30 percent of the data as test data for 100 times.

Step 5. Evaluating the superiority of the optimum model:In order to evaluate the performance of the optimum model and validate its superiority, it is compared to other forecasting tools such as different conventional regression models in terms of MAE. 
Step 6.Efficiency estimation: Use the determined best ANN structure to model relation between inputs (IRE) and outputs (lean principles).In order to calculate efficiency scores, we have used the presented steps by Azadeh, Rouzbahman, Saberi, Valianpour, and Keramati (2013):

- Calculatethe error between the observed output value $Z_{r j}$ and optimum model output $T_{r j}$ for each output variabler and operatorjusing Equation (3).

$E_{r j}=Z_{r j}-T_{r j} \quad j=1,2, \ldots, n ; r=1,2, \ldots, 5$

- Calculate frontier function shift $\left(E_{r j}^{\prime}\right)$ for obtaining the effect of the largest positive error using Equation (4).

$E_{r j}^{\prime}=\frac{E_{r j}}{T_{r j}} \quad j=1,2, \ldots, n ; r=1,2, \ldots, 5$

- Calculate the maximum value for frontier function shift $\left(E_{v}^{\prime}\right)$ among operators (DMUs):

$E_{v}=\operatorname{Max}\left(E_{r j}^{\prime}\right) \quad j=1,2, \ldots, n ; r=1,2, \ldots, 5$

- Calculate shift $\left(S h_{r j}\right)$ for each operatorj and output $r$ as follows:

$S h_{r j}=\frac{E_{v} * T_{r j}}{T_{r v}} \quad j=1,2, \ldots, n ; r=1,2, \ldots, 5$

Where $T_{r j}$ represents the optimum model value for outputr and DMU $j$. Also, the optimum model value for outputr and DMU $v$ (which is the DMU with the maximum frontier function shift value) is represented by $T_{r v}$.

- Calculate the efficiency score $\left(F_{r j}\right)$ for output roperator jusing Equation (7).

$F_{r j}=\frac{Z_{r j}}{T_{r j}+S h_{r j}} \quad j=1,2, \ldots, n ; r=1,2, \ldots, 5$

Step 7. Sensitivity analysis: Once the efficiency scores are calculated for the conceptual model, it is possible to evaluate each factor's importance and effect by omitting it from the conceptual model and recalculating the efficiency scores. In other words, input and output variables are left out of the calculations one by one, and the efficiency scores are measured anew. The results obtained before factor elimination are compared to those yielded after elimination to find out how the factor has affected the efficiency scores. This comparison is done by using paired t-test between efficiency scores obtained before elimination of each factor and efficiency scores of after elimination. 
Step 8. Validation of obtained results:Here, the efficiency scores of the DMUs are calculated by data envelopment analysis (DEA) method, and the results obtained by the intelligent algorithm are compared to those yielded by DEA. The results attained by the algorithm are validated in case they are consistent with those of DEA. To this end, first the optimum DEA model should be selected. To do so, first, different DEA models are run and compared with each other to see if model selection influences the results. In this study, CCR input-oriented, CCR output-oriented, BCC input-oriented, and BCC output-oriented model are run. Spearman's rank-order correlation is calculated between each pair of the models. Rejection of the null hypothesis for correlation with a large coefficient shows that the results obtained by different models are similar and thus model selection does not affect the results. However, to choose a single model, a noise is inserted to the data, and the model with the lowest sensitivity to this noise is selected as the optimum model. This is also examined by Spearman's correlation test between the results related to each model before and after noise insertion. The model with the highest coefficient is selected as the preferred DEA model.Mathematical programming model of considered DEA models including BCC-Input oriented, BCC-Output oriented, CCR-Output oriented and CCR-Input oriented are presented as follows:

- CCR (Charnes, Cooper and Rhodes, 1978) DEA model

Presented mathematical model evaluates the relative efficiencies of $n$ DMUs $(j=$ $1,2, \ldots, n)$ by minimizing the input variables while the outputs are considered constant. Each DMU contains $m$ input variables $\left(x_{1 j}, x_{2 j}, \ldots, x_{m j}\right)$ and $\mathrm{s}$ output variables $\left(y_{1 j}, y_{2 j}, \ldots, y_{s j}\right)$. The input-oriented CCR model is presented in Equation 8.

$\operatorname{Min} \theta$

s.t. $\quad \theta x_{i 0} \geq \sum_{j=1}^{n} \lambda_{j} x_{i j}, \quad i=1, \ldots, m$

$$
\begin{gathered}
y_{r 0} \leq \sum_{\substack{j=1 \\
\lambda_{j} \geq 0 j=1, \ldots, n}}^{n} \lambda_{j} y_{r j}, \quad r=1, \ldots, s \\
\text {. }
\end{gathered}
$$

- The output-oriented model is obtained by maximizing output variables while input variables are considered constant. The output-oriented CCR model is presented in Equation 9. 
$\operatorname{Max} \theta$

$$
\begin{gathered}
\text { s.t. } \quad x_{i 0} \geq \sum_{j=1}^{n} \lambda_{j} x_{i j}, \quad i=1, \ldots, m \\
\theta y_{r 0} \leq \sum_{j=1}^{n} \lambda_{j} y_{r j}, \quad r=1, \ldots, s \\
\lambda_{j} \geq 0 j=1, \ldots, n
\end{gathered}
$$

- BCC (Banker et al. 1984) DEA model

BCC model is similar to CCR model while one more constraint is added, which ensures that the summation of $\lambda_{j}$ is equal to 1 . Output-oriented BCC model is presented in Equation 10.

$\operatorname{Max} \theta$

s.t. $\quad x_{i 0} \geq \sum_{j=1}^{n} \lambda_{j} x_{i j}, \quad i=1, \ldots, m$

$\theta y_{r 0} \leq \sum_{j=1}^{n} \lambda_{j} y_{r j}, \quad r=1, \ldots, s$

$\sum_{j=1}^{n} \lambda_{j}=1$

$\lambda_{j} \geq 0, \quad j=1, \ldots, n$

- Input-oriented BCC model is presented in Equation 11.

Min $\theta$

s.t. $\theta x_{i 0} \geq \sum_{j=1}^{n} \lambda_{j} x_{i j}, \quad i=1, \ldots, m$

$y_{r 0} \leq \sum_{j=1}^{n} \lambda_{j} y_{r j}, \quad r=1, \ldots, s$

$\sum_{j=1}^{n} \lambda_{j}=1$

$\lambda_{j} \geq 0, \quad j=1, \ldots, n$

After determination of the optimum DEA model, the model is run based on the conceptual model, and the efficiency scores are calculated. The high consistency between efficiency scores yielded by optimum DEA model and those yielded by proposed algorithm, validates the algorithm results.

\section{Case study}


In this section, we apply the algorithm to a pipe manufacturer in Iran as our real-world case study with the aim of investigating its performance from the viewpoints of IRE and lean.

\subsection{Data collection}

Step1.IRE factors are considered as inputs and lean factors as outputs of the conceptual model. Required data collected by questionnaires which are designed and divided among staff of pipe factory, 75 operators answered the questionnaire. The respondents could select a number from among 1 to 10 for answering the questionnaire. The questionnaire designed and used in this study is presented in Table I in Appendix I.

\subsection{Reliability and validity of the questionnaire}

Step 2.The reliability of gathered data from questionnaire was analyzed by calculating Cronbach's alpha using SPSS software, related results are presented in Table 1. First, standard questionnaires which contained queries about the factors of both IRE and lean were distributed among personnel.

Table 1. Reliability of the collected data

\begin{tabular}{|c|c|c|}
\hline & Factors & Cronbach's Alpha \\
\hline \multirow{10}{*}{$\begin{array}{l}\text { Resilience } \\
\text { Engineering } \\
\text { Factors }\end{array}$} & "Reporting culture” & 0.842 \\
\hline & “Learning” & 0.742 \\
\hline & “Awareness” & 0.647 \\
\hline & “Top management commitment” & 0.842 \\
\hline & “Flexibility” & 0.727 \\
\hline & "Self-organization" & 0.694 \\
\hline & “Preparedness” & 0.801 \\
\hline & “Teamwork” & 0.783 \\
\hline & “Redundancy” & 0.728 \\
\hline & “Fault-tolerant" & 0.786 \\
\hline \multirow{5}{*}{$\begin{array}{c}\text { Lean } \\
\text { Factors }\end{array}$} & “Customer orientation” & 0.942 \\
\hline & “Reduce waste” & 0.762 \\
\hline & "Standard product" & 0.862 \\
\hline & “Pull system” & 0.941 \\
\hline & “Task management” & 0.883 \\
\hline
\end{tabular}

\subsection{Efficiency score estimation using ANN}

Step 3 and 4. In this paper we have used three different ANN models for function estimation. In order to find the best prediction structure of ANN models, different structures of estimation networks including ANN-MLP, RBF and ANFIS models are considered, and their related MAE is calculated. Since there might be a noise in ANN results, each model has been run 20 times and the average of gathered MAE is considered for models' comparison. The architecture of the ANN-RBF, ANN-MLP and ANFIS models and their related MAE values are presented in Tables 2, 3 and 4 respectively. 
Table 2. Different structures of RBF considered for comparison, and their related MAE

\begin{tabular}{ccccccc|c|c|}
\hline $\begin{array}{c}\text { Model } \\
\text { No. }\end{array}$ & Spread & $\begin{array}{c}\text { Maximum } \\
\text { Number of } \\
\text { Neurons }\end{array}$ & $\begin{array}{c}\text { Customer } \\
\text { Orientation }\end{array}$ & $\begin{array}{c}\text { Reduce } \\
\text { Waste }\end{array}$ & $\begin{array}{c}\text { Standard } \\
\text { Product }\end{array}$ & $\begin{array}{c}\text { Pull } \\
\text { System }\end{array}$ & $\begin{array}{c}\text { Task } \\
\text { Management }\end{array}$ \\
\hline 1 & 2 & 25 & 0.130 & $\mathbf{0 . 1 1 8}$ & 0.142 & 0.186 & $\mathbf{0 . 1 0 2}$ \\
2 & 4 & 25 & $\mathbf{0 . 1 1 1}$ & 0.134 & 0.148 & 0.144 & 0.138 \\
3 & 0.5 & 30 & 0.186 & 0.184 & 0.204 & 0.228 & 0.182 \\
4 & 2 & 15 & 0.193 & 0.157 & 0.166 & 0.155 & 0.153 \\
5 & 4 & 12 & 0.119 & 0.194 & 0.152 & 0.164 & 0.133 \\
6 & 3 & 20 & 0.084 & 0.157 & $\mathbf{0 . 1 2 9}$ & $\mathbf{0 . 1 3 1}$ & 0.127 \\
7 & 5 & 10 & 0.227 & 0.194 & 0.177 & 0.188 & 0.163 \\
8 & 5 & 20 & 0.112 & 0.167 & 0.164 & 0.170 & 0.148 \\
9 & 4 & 20 & 0.148 & 0.133 & 0.140 & 0.140 & 0.117 \\
10 & 3 & 15 & 0.126 & 0.172 & 0.138 & 0.159 & 0.123 \\
\hline
\end{tabular}


Table 3. Different structures of ANN-MLP considered for comparison, and related MAE

\begin{tabular}{|c|c|c|c|c|c|c|c|c|c|}
\hline \multirow[b]{2}{*}{$\mathrm{N}$} & \multirow[b]{2}{*}{ Tr. F } & \multirow[b]{2}{*}{$\begin{array}{c}\text { TF of } \\
\text { HL }\end{array}$} & \multirow[b]{2}{*}{ TO of OL } & \multirow[b]{2}{*}{ Number of neurons in HL } & \multicolumn{5}{|c|}{ MAE } \\
\hline & & & & & $\begin{array}{l}\text { Customer } \\
\text { Orientation }\end{array}$ & Reduce Waste & $\begin{array}{l}\text { Standard } \\
\text { Product }\end{array}$ & $\begin{array}{c}\text { Pull } \\
\text { System }\end{array}$ & $\begin{array}{c}\text { Task } \\
\text { Management }\end{array}$ \\
\hline 1 & LM & Logsig & Pureline & 5 & 0.2493 & 0.2028 & 0.2074 & 0.2614 & 0.2153 \\
\hline 2 & OSS & Logsig & Pureline & 12 & 0.2184 & 0.2114 & 0.2284 & 0.2556 & 0.2405 \\
\hline 3 & GD & Logsig & Pureline & 15 & 0.2008 & 0.1925 & 0.2243 & 0.2694 & 0.1922 \\
\hline 4 & BFG & Logsig & Pureline & 8 & 0.1966 & 0.1875 & 0.1861 & 0.2484 & 0.1942 \\
\hline 5 & LM & Logsig & Pureline & 30 & 0.2189 & 0.1677 & 0.2050 & 0.2433 & 0.1566 \\
\hline 6 & GDX & Logsig & Pureline & 35 & 0.2107 & 0.1994 & 0.1993 & 0.2620 & 0.2242 \\
\hline 7 & GDA & Logsig & Pureline & 45 & 0.2213 & 0.2195 & 0.2097 & 0.2598 & 0.2070 \\
\hline 8 & OSS & Logsig & Pureline & 10 & 0.1692 & 0.2114 & 0.2114 & 0.2584 & 0.2021 \\
\hline 9 & GD & tansig & Pureline & 35 & 0.1738 & 0.1922 & 0.2198 & 0.2892 & 0.1984 \\
\hline 10 & OSS & tansig & Pureline & 5 & 0.2235 & 0.1972 & 0.2052 & 0.2679 & 0.1866 \\
\hline 11 & BFG & tansig & Pureline & 7 & 0.2196 & 0.1931 & 0.1974 & 0.2393 & 0.2188 \\
\hline 12 & LM & tansig & Pureline & 10 & 0.1858 & 0.2057 & 0.2104 & 0.2453 & 0.2216 \\
\hline 13 & GDM & tansig & Pureline & 9 & 0.2184 & 0.1846 & 0.2174 & 0.2568 & 0.2183 \\
\hline 14 & OSS & tansig & Pureline & 38 & 0.2334 & 0.1805 & 0.2248 & 0.2686 & 0.2182 \\
\hline
\end{tabular}

Note. Tr. F: Training function; TF: Transfer function; HL: hidden layer; OL: Output layer; LM: Levenberg-Marquardt back propagation; BFG: quasi-Newton back propagation; GD: Gradient descent back propagation; GDA: Gradient descent with adaptive learning rule back propagation; OSS: One step secant back propagation; GDX: Gradient descent with momentum and adaptive learning rule back propagation

Table 4. Different structures of ANFIS considered for comparison and related MAE

\begin{tabular}{|c|c|c|c|c|c|c|c|c|c|c|c|c|c|c|}
\hline $\mathrm{N}$ & $\begin{array}{l}\text { Initial } \\
\text { FIS }\end{array}$ & Radius & $\begin{array}{l}\mathrm{N} \text { of } \\
\mathrm{MF}\end{array}$ & $\begin{array}{c}\mathrm{N} \text { of } \\
\text { Clusters }\end{array}$ & $\begin{array}{l}\text { Optimization } \\
\text { method }\end{array}$ & And & Or & $\begin{array}{l}\text { Implication } \\
\text { method }\end{array}$ & $\begin{array}{l}\text { Aggregation } \\
\text { method }\end{array}$ & $\begin{array}{l}\text { Customer } \\
\text { Orientation }\end{array}$ & $\begin{array}{l}\text { Reduce } \\
\text { Waste }\end{array}$ & $\begin{array}{l}\text { Standard } \\
\text { Product }\end{array}$ & $\begin{array}{c}\text { Pull } \\
\text { System }\end{array}$ & $\begin{array}{c}\text { Task } \\
\text { Management }\end{array}$ \\
\hline 1 & $\mathrm{FC}$ & - & - & 3 & $\mathrm{H}$ & prod & $\max$ & $\min$ & $\max$ & 0.100 & 0.127 & 0.142 & 0.153 & 0.113 \\
\hline 2 & FC & - & - & 6 & BP & $\min$ & probor & prod & sum & 0.116 & 0.113 & 0.157 & 0.186 & 0.097 \\
\hline 3 & $\mathrm{FC}$ & - & - & 7 & $\mathrm{H}$ & prod & probor & prod & $\max$ & 0.139 & 0.109 & 0.138 & 0.201 & 0.103 \\
\hline 4 & $\mathrm{FC}$ & - & - & 5 & $\mathrm{BP}$ & $\min$ & $\max$ & prod & sum & 0.162 & $\mathbf{0 . 0 8 0}$ & 0.162 & 0.190 & 0.121 \\
\hline 5 & GP & - & 3 & - & BP & $\min$ & probor & $\min$ & sum & 0.113 & 0.132 & 0.137 & 0.163 & 0.100 \\
\hline
\end{tabular}




\begin{tabular}{|c|c|c|c|c|c|c|c|c|c|c|c|c|c|c|}
\hline 6 & GP & - & 2 & - & $\mathrm{H}$ & prod & probor & prod & $\max$ & 0.148 & 0.141 & 0.152 & 0.188 & 0.131 \\
\hline 7 & GP & - & 2 & - & BP & $\min$ & $\max$ & $\min$ & sum & 0.114 & 0.125 & 0.169 & 0.164 & 0.114 \\
\hline 8 & GP & - & 3 & - & $\mathrm{H}$ & prod & probor & $\min$ & $\max$ & 0.124 & 0.108 & 0.182 & 0.188 & 0.102 \\
\hline 9 & SC & 0.6 & - & - & $\mathrm{H}$ & prod & $\max$ & prod & sum & 0.153 & 0.110 & 0.153 & 0.169 & 0.120 \\
\hline 10 & SC & 0.5 & - & - & $\mathrm{BP}$ & $\min$ & $\max$ & prod & $\max$ & 0.147 & 0.124 & 0.148 & 0.155 & 0.099 \\
\hline 11 & SC & 0.3 & - & - & $\mathrm{H}$ & $\min$ & probor & $\min$ & sum & 0.121 & 0.142 & 0.143 & 0.166 & 0.121 \\
\hline 12 & SC & 0.4 & - & - & $\mathrm{BP}$ & prod & probor & prod & $\max$ & 0.137 & 0.100 & 0.151 & 0.180 & 0.116 \\
\hline
\end{tabular}

Note: Input membership function for all structures: Gaussmf; Output member ship function for all structures: Linear; FIS: Fuzzy inference system; H: Hybrid; BP: BackPropagation; N: Number; MF: Membership function; FC: Fuzzy c-means (FCM) clustering; GP: Grid partition; SC: Subtractive clustering 
Step 5. Once the best model has been determined for each output, in order to examine their estimation efficiency, they are compared to three regression models, namely linear, quadratic and exponential regressions. The results are given in Table 5. Moreover, it is shown that the intelligent methods of the algorithm such as ANN-MLP, ANN-RBF and ANFIS provide better solution than conventional regression methods. This may be considered as structural validation of the proposed algorithm.

Table 5. Comparison of the best intelligent model to conventional regression methods

\begin{tabular}{cccccc}
\hline \multirow{2}{*}{ Model } & \multicolumn{5}{c}{ Mean absolute error (MAE) } \\
\cline { 2 - 6 } & $\begin{array}{c}\text { Customer } \\
\text { Orientation }\end{array}$ & $\begin{array}{c}\text { Reduce } \\
\text { Waste }\end{array}$ & $\begin{array}{c}\text { Standard } \\
\text { Product }\end{array}$ & $\begin{array}{c}\text { Pull } \\
\text { System }\end{array}$ & $\begin{array}{c}\text { Task } \\
\text { Management }\end{array}$ \\
\hline Linear regression & 0.119 & 0.102 & 0.142 & 0.141 & 0.112 \\
Quadratic regression & 0.112 & 0.108 & 0.138 & 0.136 & 0.116 \\
Exponential regression & 0.119 & 0.111 & 0.151 & 0.139 & 0.109 \\
Optimum model & 0.100 & 0.080 & 0.129 & 0.131 & 0.097 \\
Corresponding intelligent & ANFIS & ANFIS & ANN-RBF & ANN-RBF & ANFIS \\
model & Number 1 & Number 4 & Number 6 & Number 6 & Number 2 \\
Corresponding structure & Numeryyyyyy
\end{tabular}

Step 6. Table 5 shows that the optimum models enjoy the minimum MAE values and thus remain optimum. These models are then employed for measuring efficiency scores of DMUs according to the algorithm steps. The results are presented in AppendixIII.

\subsection{Sensitivity analysis}

Step 7.In this step, the way each factor of IRE and lean affect the performance is analyzed through statistical techniques. For this purpose, as mentioned in the methodology section, each factoris eliminated from the calculations, and the efficiency scores are measured anew. After obtaining the efficiency scores related to elimination of each factor, it is possible to compare mean of efficiencies before factor's elimination with related efficiency scores to elimination of each factor. Since the decision making units (DMUs) are similar in both experiments, paired t-test is employed. Paired t-test is then used to compare the results related to the original situation to those obtained after eliminating each factor. In this test, null hypothesis is $\mu_{1}=\mu_{2}$ and alternative is $\mu_{1}>\mu_{2}$ or $\mu_{1}<\mu_{2}$ which indicates that after elimination of each factor, the efficiency scores are significantly changed or not. The related results for sensitivity analysis are presented in Table 6 .

Table 6. Analysis of the factors' effects 


\begin{tabular}{|c|c|c|c|c|}
\hline & Factors & $\begin{array}{c}\text { Two-tailed } \\
\text { paired t-test p- } \\
\text { value }\end{array}$ & $\begin{array}{c}\text { One-tailed } \\
\text { paired t-test } \\
\text { result }\end{array}$ & Effect \\
\hline \multirow{5}{*}{ 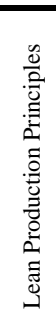 } & No Customer Orientation & 0.076 & - & Ineffective \\
\hline & No Reduce Waste & 0.300 & - & Ineffective \\
\hline & No Standard Product & 0.082 & - & Ineffective \\
\hline & No Pull System & 0.000 & $\mu_{i}>\mu_{F}$ & Negative \\
\hline & No Task Management & 0.000 & $\mu_{i}<\mu_{F}$ & Positive \\
\hline \multirow{10}{*}{ 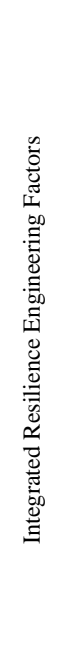 } & No Reporting culture & 0.000 & $\mu_{i}<\mu_{F}$ & Positive \\
\hline & No Learning & 0.000 & $\mu_{i}<\mu_{F}$ & Positive \\
\hline & No Awareness & 0.000 & $\mu_{i}<\mu_{F}$ & Positive \\
\hline & No Top management commitment & 0.000 & $\mu_{i}<\mu_{F}$ & Positive \\
\hline & No Flexibility & 0.067 & - & Ineffective \\
\hline & No Self-organization & 0.083 & - & Ineffective \\
\hline & No Preparedness & 0.000 & $\mu_{i}<\mu_{F}$ & Positive \\
\hline & No Teamwork & 0.000 & $\mu_{i}<\mu_{F}$ & Positive \\
\hline & No Redundancy & 0.220 & - & Ineffective \\
\hline & No Fault-tolerant & 0.000 & $\mu_{i}>\mu_{F}$ & Negative \\
\hline
\end{tabular}

The obtained results in Table 6 determine the current state of each factor in the considered system. Thus, system weaknesses and strengths from the integrated IRE and Lean production are identified and addressed. The hypothesis of equal means has been accepted for certain factors including “customer orientation”, “reduce waste”, “flexibility”, "Standard Product", "Self-Organization" and "Redundancy". Therefore, the stated factors are implemented effectively in the system. However, they should not be considered as strength or weakness of the system under study (ineffective). The null hypothesis is rejected for the rest of considered factors. This shows eliminating these factors make significant changes on efficiency scores. Mean efficiencies are compared before and after factor elimination to find out if the impact of the interested factor is positive or negative? It shows the negative impact of the factor if mean efficiency has been increased such as "pull system" and "fault-tolerant". It shows the positive impact of the factor if mean efficiency has been decreased such as "task management”, “reporting culture”, “learning”, “awareness”, “top management commitment”, "preparedness" and "teamwork". According to Table 6, among the factors of lean production, "pull system” and "task management” have the most negative and positive effects on performance. 
The results of this study can be extended to similar manufacturing environments. Moreover, the same approach and analogy may be used for performance improvement of integrated lean production and resilience engineering. It is an intelligent practical approach for decision-makers and managers of identical manufacturing and production units.

\subsubsection{Weight calculation}

Weights of each factor is calculated based on results of sensitivity analysis. In order to calculate weights, the percentage difference between correlation of omitted factors and full factor condition is computed. Figure 2 presents the calculated weights of IRE and lean production factors. Management can use the results of this section to prioritize the continuous improvement plans.

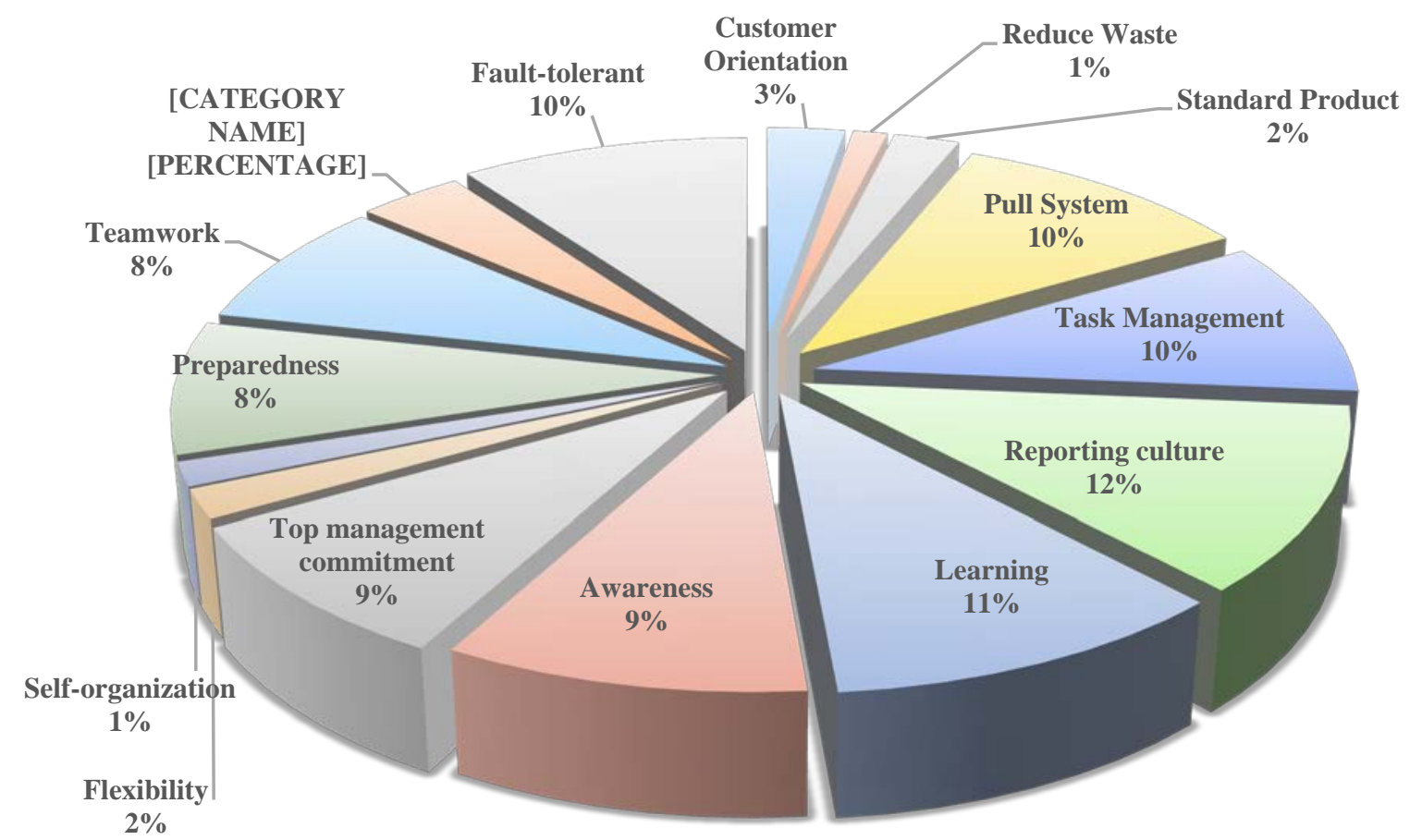

Figure 2. Weight of each considered factor in the evaluation

\subsection{Verification and validation}

Step 8. In order to validate the results obtained by the algorithm, DEA method is used to measure the efficiency scores as well, and the results yielded by the two approaches are compared with each other. As mentioned in the methodology, first the optimum DEA should be selected. For this purpose,20\% noise is employed on five random DMUs. Spearman's correlation results between efficiency scores before and after noise are presented in Table 7. 
Table 7. Noise test results for determining the optimum DEA model

\begin{tabular}{|c|c|c|c|c|c|c|c|}
\hline \multicolumn{2}{|c|}{ BCC-output oriented } & \multicolumn{2}{|c|}{ BCC-input oriented } & \multicolumn{2}{|c|}{ CCR-output oriented } & \multicolumn{2}{|c|}{ CCR-input oriented } \\
\hline Before noise & After noise & Before noise & After noise & Before noise & After noise & Before noise & After noise \\
\hline 1 & 1 & 1 & 1 & 0.96717423 & 0.9715926 & 1.033939873 & 1.029238 \\
\hline 0.819499061 & 0.8194991 & 1.031926864 & 1.03192686 & 0.81047607 & 0.8104761 & 1.233842725 & 1.23384272 \\
\hline 1 & 1 & 0.999999999 & 1 & 0.91351142 & 0.9135114 & 1.094677067 & 1.09467707 \\
\hline 1.003900988 & 1 & 0.99826017 & 1 & 1.00174286 & 1 & 0.99826017 & 0.97346738 \\
\hline 0.931657851 & 0.9316578 & 1.147838495 & 1.14778486 & 0.85723185 & 0.8572318 & 1.166545547 & 1.16654555 \\
\hline 1 & 1 & 1 & 1 & 1 & 1 & 1 & 1 \\
\hline 0.989349912 & 0.9902381 & 0.973473483 & 0.98374375 & 0.97980137 & 0.9798014 & 1.020615029 & 1.02061503 \\
\hline 0.874722975 & 0.8858508 & 1.019268173 & 1.01124239 & 0.81115676 & 0.8111568 & 1.232807336 & 1.23280734 \\
\hline 1.000000001 & 1 & 1 & 1 & 1 & 1 & 1 & 1 \\
\hline 1 & 1 & 0.999999995 & 1.01380859 & 0.86887611 & 0.8688761 & 1.150912069 & 1.15091207 \\
\hline 0.785377354 & 0.7868349 & 1.091350022 & 1.09135002 & 0.78210313 & 0.7821031 & 1.278603754 & 1.27860375 \\
\hline 0.869816582 & 0.8698166 & 1.084181422 & 1.08418142 & 0.85279803 & 0.852798 & 1.172610587 & 1.17261059 \\
\hline 0.9375 & 0.9375 & 1.037070048 & 1.03707005 & 0.92298684 & 0.9229868 & 1.08343907 & 1.08343907 \\
\hline 0.911221956 & 0.911222 & 1.08830439 & 1.08830439 & 0.90198844 & 0.9019884 & 1.108661664 & 1.10866166 \\
\hline 0.808367356 & 0.8083674 & 1.097569515 & 1.09756951 & 0.80422859 & 0.8042286 & 1.243427572 & 1.24342757 \\
\hline 0.76680353 & 0.7668035 & 1.108108108 & 1.10810811 & 0.73578208 & 0.7357821 & 1.359098059 & 1.35909806 \\
\hline 1 & 1 & 1 & 0.95454545 & 0.8867596 & 0.8867596 & 1.127701348 & 1.12770136 \\
\hline 0.882142616 & 0.8821426 & 1.00613497 & 1.00613497 & 0.8549964 & 0.8549964 & 1.16959557 & 1.16959557 \\
\hline 1.098934634 & 1.084585 & 0.998723954 & 0.90338828 & 1 & 1.0754582 & 1 & 0.9298362 \\
\hline 1.000000001 & 1 & 1 & 1 & 1 & 1 & 1 & 1 \\
\hline 1 & 1 & 1 & 1 & 1 & 1 & 1 & 1 \\
\hline 1 & 1 & 1 & 0.99999999 & 0.8943591 & 0.8943591 & 1.118119099 & 1.1181191 \\
\hline 0.832318013 & 0.7272194 & 1.065761717 & 1.06576172 & 0.82930904 & 0.6653477 & 1.2058231 & 1.50297366 \\
\hline 1 & 1 & 1 & 1 & 1 & 1 & 0.999999994 & 1 \\
\hline 0.792835236 & 0.7942851 & 1.086209084 & 1.0860265 & 0.79264303 & 0.7937465 & 1.261601956 & 1.25984812 \\
\hline 1 & 1 & 1 & 1 & 1 & 1 & 1 & 1 \\
\hline 1 & 0.9742176 & 1 & 1.0060717 & 1 & 0.8820924 & 1 & 1.13366802 \\
\hline 0.981649218 & 0.9816492 & 1.019422395 & 1.01942239 & 0.9788315 & 0.9788315 & 1.021626295 & 1.0216263 \\
\hline 1 & 1 & 1 & 1 & 0.92554648 & 0.9257018 & 1.080442762 & 1.08026148 \\
\hline 1 & 1 & 0.999999997 & 1.00289491 & 1 & 1 & 1 & 1 \\
\hline 1.000000005 & 1 & 1 & 1 & 1.00000001 & 1 & 0.999999996 & 1.01384921 \\
\hline 1.000000001 & 1 & 1 & 1 & 1 & 1 & 1 & 1 \\
\hline 0.844601525 & 0.8446015 & 1.086905951 & 1.08642385 & 0.83056137 & 0.8305614 & 1.204004956 & 1.20400496 \\
\hline 0.889976233 & 0.8899762 & 1.097527019 & 1.09703056 & 0.8607552 & 0.8607552 & 1.161770503 & 1.1617705 \\
\hline 1.000000001 & 1 & 1 & 1 & 1 & 1 & 1 & 1 \\
\hline 1 & 1 & 1 & 1 & 1 & 1 & 1 & 1 \\
\hline 0.822851317 & 0.8228513 & 1.069284331 & 1.05037826 & 0.81988811 & 0.8198881 & 1.219678626 & 1.21967863 \\
\hline 1 & 1 & 1 & 1 & 0.97542743 & 0.9754274 & 1.025191592 & 1.02519159 \\
\hline 0.926339542 & 0.9299844 & 1.043024772 & 1.04302477 & 0.91055169 & 0.9207083 & 1.098235292 & 1.08612031 \\
\hline 0.756075475 & 0.7560755 & 1.13330244 & 1.13330244 & 0.74617586 & 0.7461759 & 1.340166636 & 1.34016664 \\
\hline 0.923003709 & 0.9230037 & 1.027197669 & 1.01973684 & 0.87962436 & 0.8796244 & 1.136848916 & 1.13684892 \\
\hline 1 & 1 & 1.147633936 & 1.14763394 & 0.87135799 & 0.871358 & 1.147633936 & 1.14763393 \\
\hline 0.956971256 & 0.9569713 & 1.031791763 & 1.03179176 & 0.95636824 & 0.9563682 & 1.045622342 & 1.04562234 \\
\hline
\end{tabular}




\begin{tabular}{|c|c|c|c|c|c|c|c|}
\hline 1 & 1 & 1 & 1 & 1 & 1 & 1 & 1 \\
\hline 1.001546391 & 1.0015464 & 0.99867021 & 0.99867021 & 0.99766053 & 0.9976605 & 1.002344965 & 1.00234496 \\
\hline 0.770384727 & 0.771287 & 1.093838642 & 1.09383864 & 0.76316855 & 0.763705 & 1.310326534 & 1.30940615 \\
\hline 0.916836142 & 0.9168361 & 1.159720512 & 1.15972052 & 0.82932174 & 0.8293217 & 1.205804641 & 1.20580463 \\
\hline 0.76303681 & 0.7630368 & 1.131240521 & 1.13060786 & 0.73943235 & 0.7394324 & 1.352388767 & 1.35238877 \\
\hline 0.843516593 & 0.8435166 & 1.029939347 & 1.02993935 & 0.83749764 & 0.8397834 & 1.194033216 & 1.19078331 \\
\hline 0.895523697 & 0.8955237 & 1.043750263 & 1.04375026 & 0.89023053 & 0.8906014 & 1.123304545 & 1.12283676 \\
\hline 1 & 1 & 1 & 1 & 1 & 1 & 1 & 1 \\
\hline 1 & 1 & 1 & 1 & 0.99746127 & 0.9974613 & 1.002545192 & 1.0025452 \\
\hline 0.8258185 & 0.8258185 & 1.048917906 & 1.04891791 & 0.82047628 & 0.8204763 & 1.218804273 & 1.21880427 \\
\hline 0.772525486 & 0.7725255 & 1.078947368 & 1.07894737 & 0.75515594 & 0.7551559 & 1.324229801 & 1.3242298 \\
\hline 0.947392847 & 0.9572381 & 1.012390483 & 1.0129394 & 0.91226158 & 0.9122616 & 1.096176824 & 1.09617682 \\
\hline 1 & 1 & 1 & 1 & 0.83675568 & 0.8367557 & 1.195637179 & 1.19509198 \\
\hline 1 & 1 & 1 & 1 & 1 & 1 & 1 & 1 \\
\hline 1 & 1 & 1 & 1 & 1 & 1 & 1 & 1 \\
\hline 1 & 1 & 0.9999999997 & 1 & 0.9721992 & 0.976701 & 1.028595785 & 1.02445405 \\
\hline 0.801400168 & 0.8014002 & 1.108019103 & 1.1080191 & 0.79911726 & 0.7991173 & 1.251380811 & 1.25138081 \\
\hline 0.770825373 & 0.7708254 & 1.163280111 & 1.16328012 & 0.76063997 & 0.76064 & 1.314682429 & 1.31468243 \\
\hline 1 & 1 & 1 & 0.99999999 & 0.94696965 & 0.9469697 & 1.056000051 & 1.05600005 \\
\hline 0.771471247 & 0.7714712 & 1.117250439 & 1.11714543 & 0.74514503 & 0.7459164 & 1.34202062 & 1.34063287 \\
\hline 1 & 1 & 1 & 1 & 1 & 1 & 1 & 1 \\
\hline 1 & 1 & 1 & 1 & 1 & 1 & 1 & 1 \\
\hline 1 & 1 & 1 & 1 & 1 & 1 & 1 & 1 \\
\hline 0.901711912 & 0.9017119 & 1.039257105 & 1.0392571 & 0.89605832 & 0.8960583 & 1.115998785 & 1.11599879 \\
\hline 1 & 1 & 0.9999999999 & 0.999999999 & 1 & 1 & 1 & 1 \\
\hline 0.893453771 & 0.8934538 & 1.068062591 & 1.06806259 & 0.89170635 & 0.8917063 & 1.121445426 & 1.12144543 \\
\hline 1 & 1 & 1 & 1 & 1 & 1 & 1 & 1 \\
\hline 0.86040725 & 0.8604072 & 1.08345153 & 1.08079763 & 0.85875323 & 0.8587532 & 1.164478878 & 1.16447888 \\
\hline 0.893750874 & 0.8937509 & 1.108108113 & 1.10810811 & 0.83204236 & 0.8320424 & 1.201861883 & 1.20186188 \\
\hline 1 & 1 & 1 & 1 & 1 & 1 & 1 & 1 \\
\hline 0.941157759 & 0.9411578 & 1.073824713 & 1.07382471 & 0.90723439 & 0.9072344 & 1.102250986 & 1.10225099 \\
\hline 0.892547852 & 0.8926603 & 1.022891514 & 1.02289151 & 0.86121911 & 0.8612191 & 1.161144693 & 1.1611447 \\
\hline \multicolumn{8}{|c|}{ Spearman's Correlation Coefficient } \\
\hline \multicolumn{2}{|c|}{0.9895181} & \multicolumn{2}{|c|}{0.9706354} & \multicolumn{2}{|c|}{0.9626219} & \multicolumn{2}{|c|}{0.94783941} \\
\hline
\end{tabular}

As indicated in Table 7, BCC-Input oriented model has the least sensitivity to created noise. Therefore, it is selected as the optimum DEA model to validate the obtained results.After determination of the optimum DEA model, the model is run based on the conceptual model, and the efficiency scores are calculated. The high consistency between efficiency scores yielded by optimum DEA model and those yielded by proposed algorithm, validates the algorithm results. The value of Spearman correlation coefficient between the ranks of DMUs obtained by the algorithm and those by DEA optimum model is equal to 0.873 (p-value $=0.012$ ) which shows that the results are similar, and thus the results obtained 
by the algorithm are valid.The efficiency scores obtained by the optimum DEA model and the proposed algorithmare presented in Table 8.

Table 8. Verification and validation results

\begin{tabular}{|c|c|c|}
\hline \multirow{2}{*}{ DMU } & \multicolumn{2}{|c|}{ Efficiency Score } \\
\hline & Proposed Algorithm & Optimum DEA \\
\hline 1 & 0.78204 & 1 \\
\hline 2 & 0.68031 & 0.8194991 \\
\hline 3 & 0.79237 & 1 \\
\hline 4 & 0.83238 & 1.003901 \\
\hline 5 & 0.74238 & 0.9316579 \\
\hline 6 & 0.79576 & 1 \\
\hline 7 & 0.74894 & 0.9893499 \\
\hline 8 & 0.68031 & 0.874723 \\
\hline 9 & 0.76397 & 1 \\
\hline 10 & 0.78267 & 1 \\
\hline 11 & 0.59845 & 0.7853774 \\
\hline 12 & 0.54724 & 0.8698166 \\
\hline 13 & 0.69458 & 0.9375 \\
\hline 14 & 0.66830 & 0.911222 \\
\hline 15 & 0.56545 & 0.8083674 \\
\hline 16 & 0.52388 & 0.7668035 \\
\hline 17 & 0.82723 & 1 \\
\hline 18 & 0.63483 & 0.8821426 \\
\hline 19 & 0.83746 & 1.0989346 \\
\hline 20 & 0.82373 & 1 \\
\hline 21 & 0.67834 & 1 \\
\hline 22 & 0.78347 & 1 \\
\hline 23 & 0.58940 & 0.832318 \\
\hline 24 & 0.72383 & 1 \\
\hline 25 & 0.54992 & 0.7928352 \\
\hline 26 & 0.90273 & 1 \\
\hline 27 & 0.89294 & 1 \\
\hline 28 & 0.73873 & 0.9816492 \\
\hline 29 & 0.82738 & 1 \\
\hline 30 & 0.79835 & 1 \\
\hline 31 & 0.74382 & 1 \\
\hline 32 & 0.80237 & 1 \\
\hline 33 & 0.60168 & 0.8446015 \\
\hline 34 & 0.64706 & 0.8899762 \\
\hline 35 & 0.74347 & 1 \\
\hline 36 & 0.81273 & 1 \\
\hline 37 & 0.57993 & 0.8228513 \\
\hline 38 & 0.68349 & 1 \\
\hline 39 & 0.68342 & 0.9263395 \\
\hline
\end{tabular}




\begin{tabular}{|c|c|c|}
\hline 40 & 0.60347 & 0.7560755 \\
\hline 41 & 0.72035 & 0.9230037 \\
\hline 42 & 0.77348 & 1 \\
\hline 43 & 0.65348 & 0.9569713 \\
\hline 44 & 0.75348 & 1 \\
\hline 45 & 0.86835 & 1.0015464 \\
\hline 46 & 0.52746 & 0.7703847 \\
\hline 47 & 0.69348 & 0.9168361 \\
\hline 48 & 0.58348 & 0.7630368 \\
\hline 49 & 0.64238 & 0.8435166 \\
\hline 50 & 0.67348 & 0.8955237 \\
\hline 51 & 0.76348 & 1 \\
\hline 52 & 0.79347 & 1 \\
\hline 53 & 0.69343 & 0.8258185 \\
\hline 54 & 0.63482 & 0.7725255 \\
\hline 55 & 0.70447 & 0.9473928 \\
\hline 56 & 0.68350 & 1 \\
\hline 57 & 0.70238 & 1 \\
\hline 58 & 0.79349 & 1 \\
\hline 59 & 0.72383 & 1 \\
\hline 60 & 0.55848 & 0.8014002 \\
\hline 61 & 0.52791 & 0.7708254 \\
\hline 62 & 0.75708 & 1 \\
\hline 63 & 0.52855 & 0.7714712 \\
\hline 64 & 0.76348 & 1 \\
\hline 65 & 0.79835 & 1 \\
\hline 66 & 0.75708 & 1 \\
\hline 67 & 0.67835 & 0.9017119 \\
\hline 68 & 0.78744 & 1 \\
\hline 69 & 0.65053 & 0.8934538 \\
\hline 70 & 0.75708 & 1 \\
\hline 71 & 0.61749 & 0.8604073 \\
\hline 72 & 0.65083 & 0.8937509 \\
\hline 73 & 0.75708 & 1 \\
\hline 74 & 0.69824 & 0.9411578 \\
\hline 75 & 0.64963 & 0.8925479 \\
\hline Spearman's Correlation Coefficient & \multicolumn{2}{|c|}{0.873} \\
\hline
\end{tabular}

In order to indicate the effectiveness of the proposed algorithm considering RE and lean production concepts together, the efficiency scores of RE and lean principles factors are calculated separately, and the results are compared to the conceptual model of the presented study (RE combined with lean principles). The results are presented in Table 9. 
Table 9. Superiority of combined RE and lean production versus each one separately

\begin{tabular}{|c|c|c|c|}
\hline Factors & Mean of Efficiencies & T-test Result & $\begin{array}{c}\text { Comparison between } \\
\text { mean of efficiencies }\end{array}$ \\
\hline $\begin{array}{c}\text { Combined RE and Lean } \\
\text { Principles }\end{array}$ & $\mu_{\text {Combined }}=0.7070$ & - & - \\
\hline RE Factors & $\mu_{R E}=0.6947$ & P-Value $=0.327$ & $\mu_{\text {Combined }}>\mu_{R E}$ \\
\hline Lean Principles & $\mu_{\text {Lean }}=0.6583$ & P-Value $=0.000$ & $\mu_{\text {Combined }}>\mu_{\text {Lean }}$ \\
\hline
\end{tabular}

As shown in Table 9, considering both concepts together provide higher efficiency scores for DMUs which validates the conceptual model of the presented study.

\section{Conclusions and directions for future research}

The goal of this paper was to conduct performance assessment from RE and lean points of view points in a pipe factory. Required data were gathered by distributing standard questionnaires among the staff. Thereafter, an adaptive intelligent flexible algorithm composedof various artificial neural networks including MLP, RBF and ANFIS as well as robust mathematical and statistical methods was used for performance assessment of the organization. IRE and lean factors were considered as input and output variables of the model, respectively. After determining the best estimation structure for each output, they were used in the algorithm to calculate decision making units (DMUs) efficiency scores. Subsequently, data envelopment analysis (DEA) method was used to validate the results obtained by the algorithm. In a significant phase of the algorithm, the way each factor of IRE and lean production affected the performance were analyzed statistically. For this purpose, each of the input and output factors were eliminated from the calculations and the efficiency scores were measure anew. The results were then compared to each other by paired t-test. According to the results obtained, after elimination of customer orientation, reduce waste and standard product, and also flexibility, self-organization and redundancy average efficiency gains $\left(\mu_{2}\right)$ is equal to the average efficiency $\left(\mu_{1}\right)$ before elimination of the stated factors $\left(\mu_{1}=\right.$ $\mu_{2}$ ). Therefore, these factors have not a significant impact on organizational efficiency. On the other hand, by eliminating reporting culture, learning, awareness, top management commitment, preparedness, and teamwork, and also task management, average efficiency $\left(\mu_{2}\right)$ is less than the average efficiency $\left(\mu_{1}\right)$ before elimination of the stated factors $\left(\mu_{1}>\mu_{2}\right)$. This means, with elimination of these factors organizational efficiency diminishes, and thus these factors have positive impact on organizational efficiency. Also, according to the results, with elimination of pull system and Fault-tolerant, efficiency increases $\left(\mu_{1}<\mu_{2}\right)$, since the 
elimination of these two factors increased efficiency, so these two factors have negative impact on organizational efficiency. The management could use these results to perform corrective actions on negative factors as well as use positive factors for benchmarking. MATLAB V.2015 is used and executed for determining the optimal structures of neural networks (parameter tuning). The codes used for RBF, MLP and ANFIS are presented in Appendix II. Microsoft Excel is used for calculating efficiency scores using mathematical algorithm. Statistical methods such as paired t-test and Cronbach's Alpha are accomplished by SPSS v. 21. DEA is executed and solved by Auto Assess (Azadeh et al, 2014). Auto Assess is capable of executing and solving various DEA, fuzzy DEA, principle component analysis (PCA) and numerical taxonomy (NT).

\section{Acknowledgements}

This study was supported by a grant from University of Tehran (No. 8106013/1/20). The authors are grateful for the support provided by the College of Engineering, University of Tehran, Tehran, Iran. This study was also supported by a grant from the Iran National Science Foundation (No. 94002128). The authors are grateful for the financial support provided by the Iran National Science Foundation.

\section{References}

Aqlan, F., \& Ali, E. M. (2014). Integrating lean principles and fuzzy bow-tie analysis for risk assessment in chemical industry. Journal of Loss Prevention in the Process Industries, 29, 3948.

Azadeh, A., Ghaderi, S., Anvari, M., \& Saberi, M. (2007). Performance assessment of electric power generations using an adaptive neural network algorithm. Energy Policy, 35(6), 3155-3166.

Azadeh, A., Rouzbahman, M., Saberi, M., Valianpour, F., \& Keramati, A. (2013). Improved prediction of mental workload versus HSE and ergonomics factors by an adaptive intelligent algorithm. Safety science, 58, 59-75.

Azadeh, A., Salehi, V., Arvan, M., \& Dolatkhah, M. (2014). Assessment of resilience engineering factors in high-risk environments by fuzzy cognitive maps: A petrochemical plant. Safety science, 68, 99-107.

Azadeh, A., Salehi, V., Ashjari, B., \& Saberi, M. (2014). Performance evaluation of integrated resilience engineering factors by data envelopment analysis: The case of a petrochemical plant. Process safety and environmental protection, 92(3), 231-241.

Banker, R. D., Charnes, A., \& Cooper, W. W. (1984). Some models for estimating technical and scale inefficiencies in data envelopment analysis. Management science, 30(9), 1078-1092.

Bashiri, M., Farshbaf-Geranmayeh, A., \& Mogouie, H. (2013). A neuro-data envelopment analysis approach for optimization of uncorrelated multiple response problems with smaller the better type controllable factors. Journal of Industrial Engineering International, 9(1), 30.

Brandao de Souza, L. (2009). Trends and approaches in lean healthcare. Leadership in Health Services, 22(2), 121-139.

Clegg, C. W. (2000). Sociotechnical principles for system design. Appl Ergon, 31(5), 463-477.

Dinh, L. T., Pasman, H., Gao, X., \& Mannan, M. S. (2012). Resilience engineering of industrial processes: Principles and contributing factors. Journal of Loss Prevention in the Process Industries, 25(2), 233-241. 
Fliedner, G. (2008). Sustainability: a new lean principle. Paper presented at the Proceedings of the 39th annual meeting of the decision sciences institute, Baltimore, Maryland.

Frangopol, D. M., \& Curley, J. P. (1987). Effects of damage and redundancy on structural reliability. Journal of Structural Engineering, 113(7), 1533-1549.

Govindan, K., Azevedo, S., Carvalho, H., \& Cruz-Machado, V. (2015). Lean, green and resilient practices influence on supply chain performance: interpretive structural modeling approach. International Journal of Environmental Science and Technology, 12(1), 15-34.

Haimes, Y. Y. (2009). On the Complex Definition of Risk: A Systems

RBßsked Approach analysis, 29(12), 1647-1654.

Hansson, L., Herrera, I. A., Kongsvik, T., \& Solberg, G. (2009). Applying the resilience concept in practice: A case study from the oil and gas industry. Safety, Reliability and Risk Analysis: Theory, Methods and Applications, 4.

Hegde, S., Wreathall, J., Hettinger, A. Z., Fairbanks, R. J., Wears, R. L., \& Bisantz, A. M. (2014). Towards the Development of a Resilience Engineering Tool to Improve Patient Safety The RETIPS Approach. Paper presented at the Proceedings of the Human Factors and Ergonomics Society Annual Meeting.

Hicks, C., McGovern, T., Prior, G., \& Smith, I. (2015). Applying lean principles to the design of healthcare facilities. International Journal of Production Economics.

Hines, P., Holweg, M., \& Rich, N. (2004). Learning to evolve: a review of contemporary lean thinking. International Journal of Operations \& Production Management, 24(10), 994-1011.

Hollnagel, E., Braithwaite, J., \& Wears, R. L. (2013). Resilient health care: Resilient Health Care: Ashgate.

Hollnagel, E., Woods, D. D., \& Leveson, N. (2007). Resilience engineering: Concepts and precepts: Ashgate Publishing, Ltd.

Jeffcott, S., Ibrahim, J., \& Cameron, P. (2009). Resilience in healthcare and clinical handover. Quality and Safety in Health Care, 18(4), 256-260.

Ling-Ling, F., \& Yong-Duan, S. (2010). On fault-tolerant control of dynamic systems with actuator failures and external disturbances. Acta Automatica Sinica, 36(11), 1620-1625.

Lunardini, D., Arington, R., Canacari, E. G., Gamboa, K., Wagner, K., \& McGuire, K. J. (2014). Lean principles to optimize instrument utilization for spine surgery in an academic medical center: an opportunity to standardize, cut costs, and build a culture of improvement. Spine, 39(20), 1714-1717.

Min, Y. T. (2014). The impact of lean implementation on quality and efficiency of US hospitals.

Nemeth, C., Wears, R., Woods, D., Hollnagel, E., \& Cook, R. (2008). Minding the gaps: creating resilience in healthcare. Advances in patient safety: new directions and alternative approaches, 3, 1-13.

Ng, D., Vail, G., Thomas, S., \& Schmidt, N. (2010). Applying the Lean principles of the Toyota Production System to reduce wait times in the emergency department. Cjem, 12(01), 50-57.

Ōno, T. (1988). Toyota production system: beyond large-scale production: Productivity press.

Plowman, D. A., Solansky, S., Beck, T. E., Baker, L., Kulkarni, M., \& Travis, D. V. (2007). The role of leadership in emergent, self-organization. The Leadership Quarterly, 18(4), 341-356.

Rasmussen, J., Pejtersen, A. M., \& Goodstein, L. P. (1994). Cognitive systems engineering: Wiley.

Ross, A., Anderson, J., Kodate, N., Thompson, K., Cox, A., \& Malik, R. (2014). Inpatient diabetes care: complexity, resilience and quality of care. Cognition, Technology \& Work, 16(1), 91102.

Sharma, V., Dixit, A. R., \& Qadri, M. A. (2015). Impact of lean practices on performance measures in context to Indian machine tool industry. Journal of Manufacturing Technology Management, 26(8), 1218-1242.

Shirali, G. A., Motamedzade, M., Mohammadfam, I., Ebrahimipour, V., \& Moghimbeigi, A. (2015). Assessment of resilience engineering factors based on system properties in a process industry. Cognition, Technology \& Work, 1-13.

Susilawati, A., Tan, J., Bell, D., \& Sarwar, M. (2015). Fuzzy logic based method to measure degree of lean activity in manufacturing industry. Journal of Manufacturing Systems, 34, 1-11.

Womack, J. P., \& Jones, D. T. (2010). Lean thinking: banish waste and create wealth in your corporation: Simon and Schuster. 
Woods, D. D., \& Hollnagel, E. (2006). Joint cognitive systems: Patterns in cognitive systems engineering: CRC Press.

Wreathall, J. (2006). Properties of resilient organizations: an initial view. Resilience engineering concepts and precepts. Burlington, VT: Ashgate.

\section{Appendix I:}

Table I. The questionnaire used for collecting required data

\begin{tabular}{|c|c|}
\hline Main Question & Factor \\
\hline $\begin{array}{l}\text { How do you assess management's willingness to invest, assign and } \\
\text { devote resources to improve safety precautions? }\end{array}$ & $\begin{array}{l}\text { Top Management } \\
\text { Commitment }\end{array}$ \\
\hline $\begin{array}{l}\text { How do you assess the willingness of the staff to report problems and } \\
\text { subjects in your organization? }\end{array}$ & Reporting Culture \\
\hline $\begin{array}{l}\text { Does your work environment encourage you to learn from past events in } \\
\text { order to respond effectively in future? }\end{array}$ & Learning \\
\hline $\begin{array}{l}\text { Does management allow you to be aware of system failures and its } \\
\text { consequences? }\end{array}$ & Awareness \\
\hline $\begin{array}{l}\text { Do you feel the system is actively preparing and predicting problems } \\
\text { and issues of human-machine systems? }\end{array}$ & Preparedness \\
\hline $\begin{array}{l}\text { Do you feel you are allowed to deal with failures using your natural } \\
\text { instincts, abilities and experiences? }\end{array}$ & Flexibility \\
\hline $\begin{array}{l}\text { Do arrangements come from the performances of interdependent agents } \\
\text { who change data, take actions, and adjust to react about other activities? }\end{array}$ & Self-organization \\
\hline How do you assess teamwork in your working environment? & Teamwork \\
\hline $\begin{array}{l}\text { Is there a replacement plan for each critical resource such as staff or } \\
\text { equipment in case of failure, breakdown or absence? }\end{array}$ & Redundancy \\
\hline $\begin{array}{l}\text { Do you feel the system is preserving to the specified function in the } \\
\text { presence of mistakes and faults? }\end{array}$ & Fault-tolerant \\
\hline How do you assess customer orientation in your organization? & Customer Orientation \\
\hline $\begin{array}{l}\text { Does your organization investigate each product value stream and } \\
\text { determine all non-value added steps? }\end{array}$ & Reduce Waste \\
\hline $\begin{array}{l}\text { Does your organization standardize all processes after designing most } \\
\text { efficient product flow? }\end{array}$ & Standard Product \\
\hline $\begin{array}{l}\text { Does your production system control flow of resources by replacing only } \\
\text { what has been consumed? }\end{array}$ & Pull System \\
\hline $\begin{array}{l}\text { How do you assess the ability of your organization to utilize resources } \\
\text { (such as time and information) efficiently? }\end{array}$ & Task Management \\
\hline \multicolumn{2}{|c|}{$\begin{array}{l}\text { The respondents can answer the question by continuous values between } 1 \text { to } 10: 1 \\
\text { stands for completely disagree while } 10 \text { is vice versa. }\end{array}$} \\
\hline
\end{tabular}

\section{Appendix II: MatLab Codes}

Table II. Multi-layer perceptron MatLab code

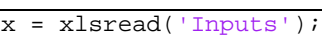




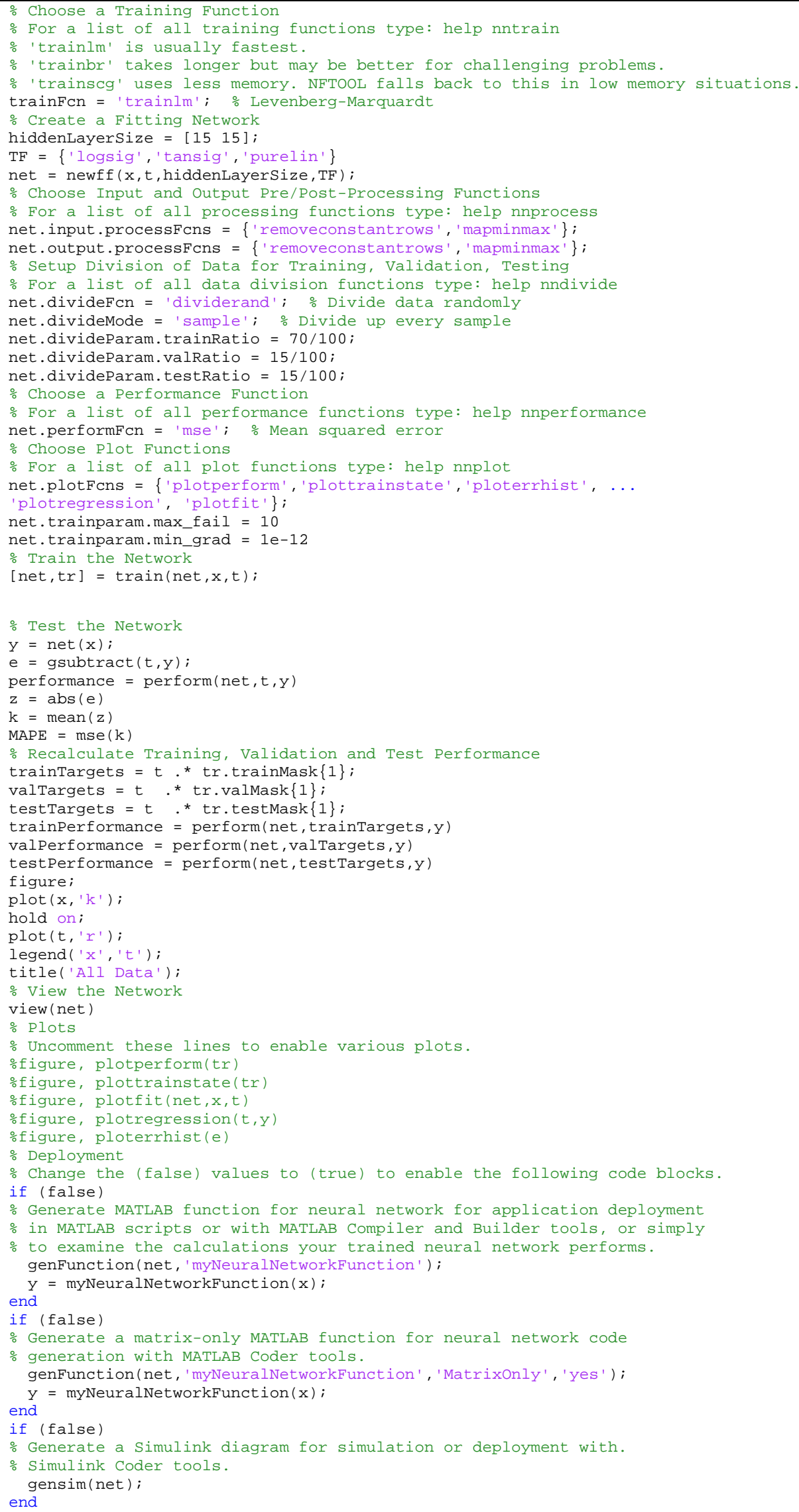


Table III. Radial basis function MatLab code

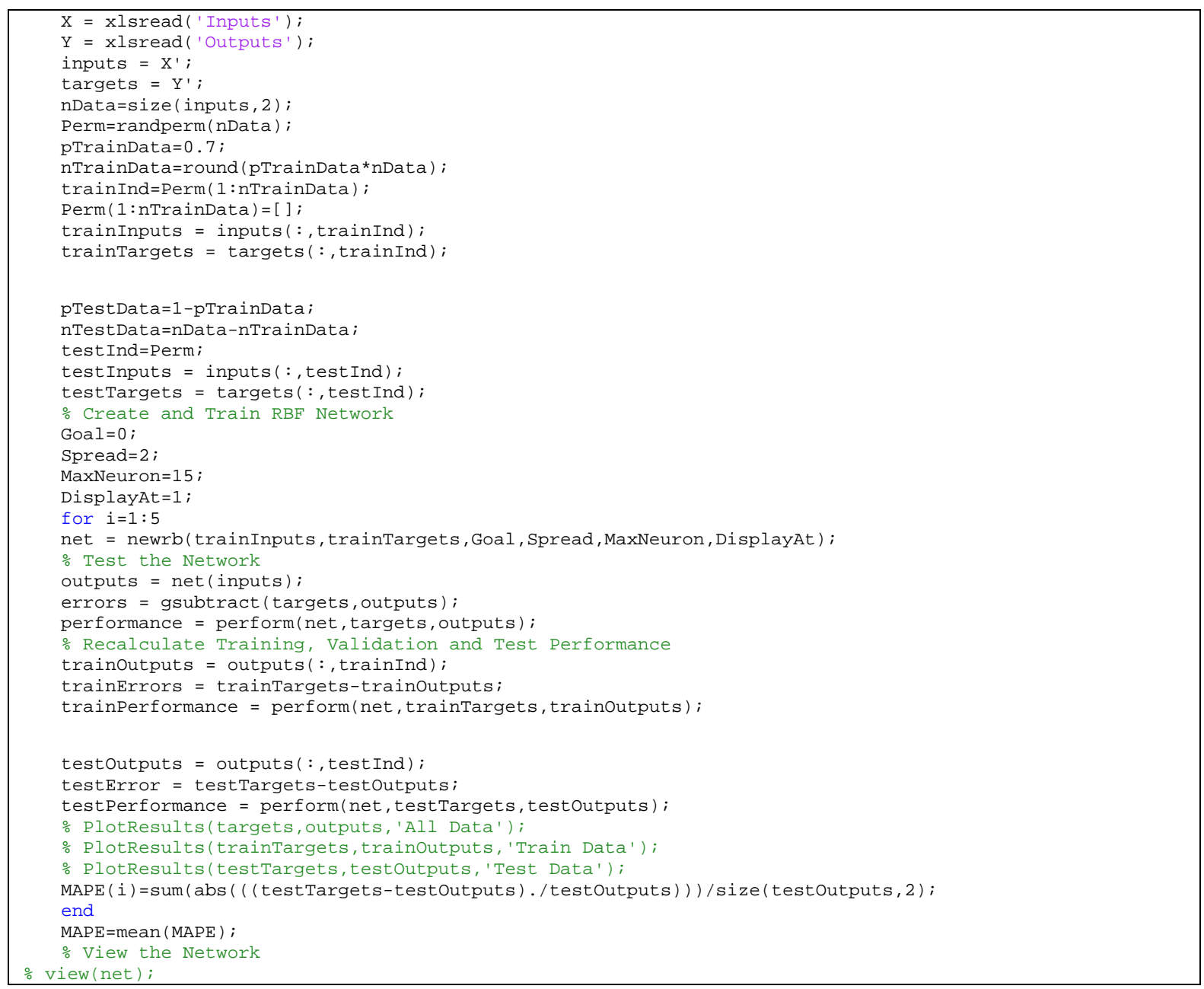

Table IV. Adaptive neuro-fuzzy inference system MatLab code

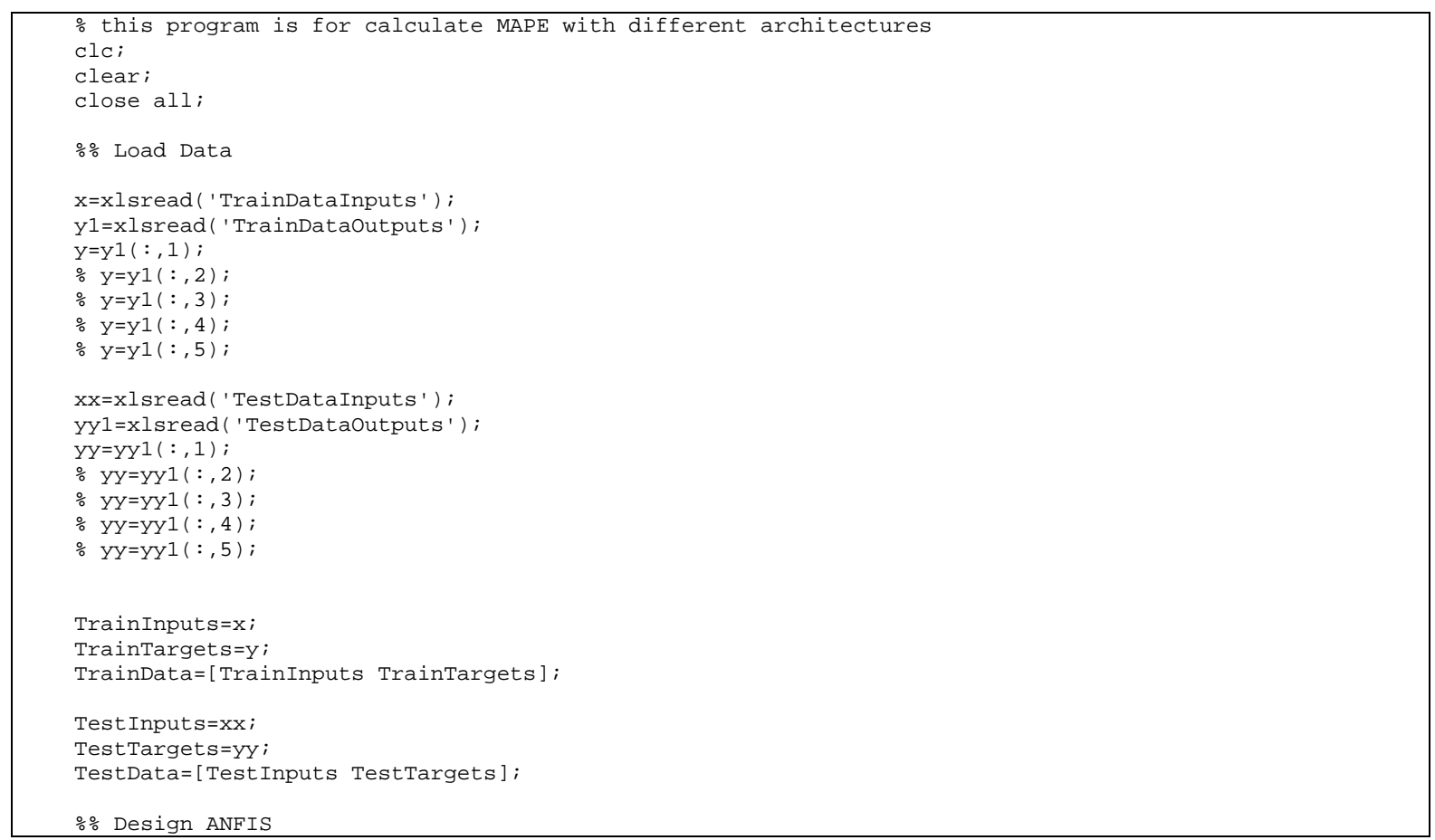




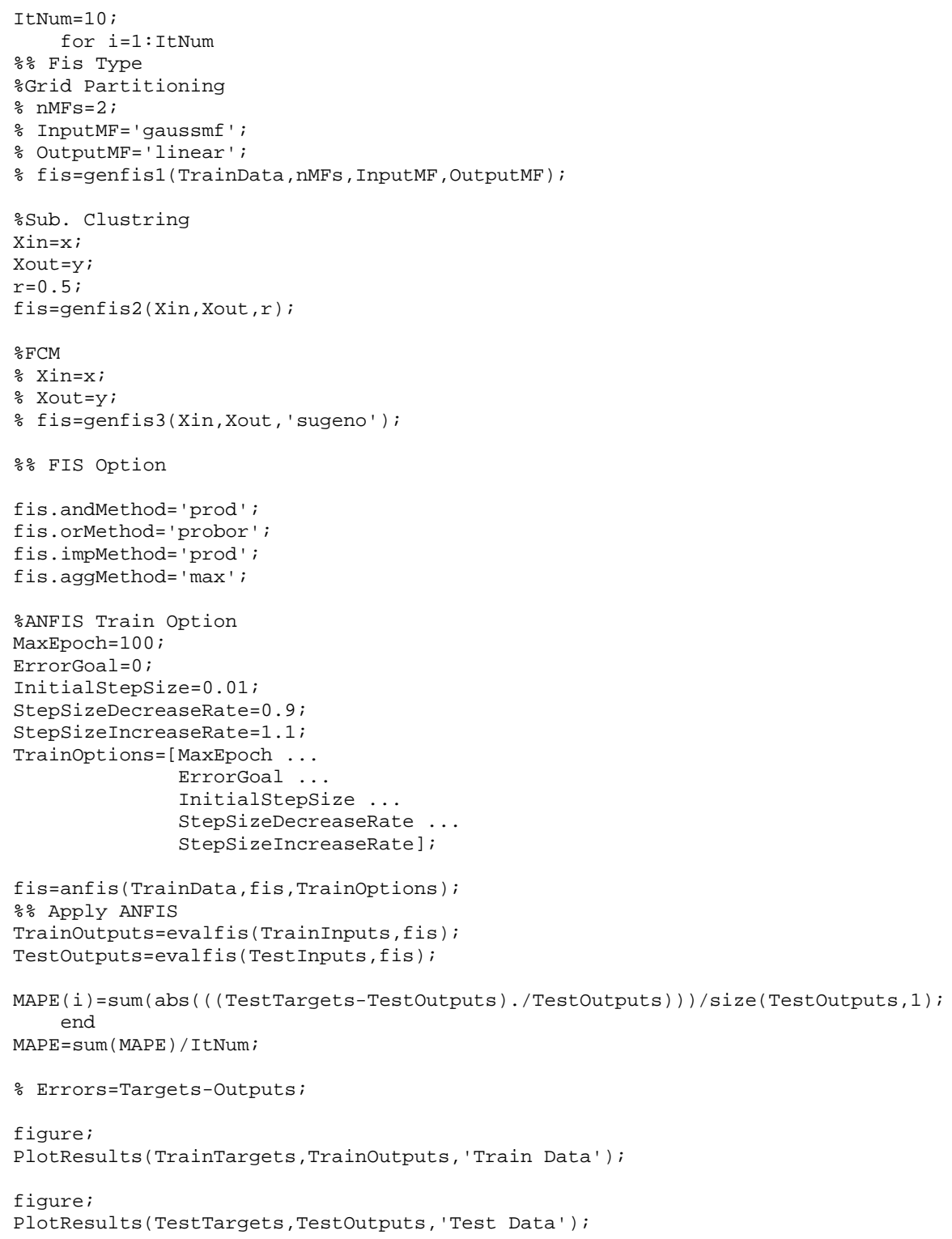

\section{Appendix III:}

Table V. Efficiency scores obtained by the algorithm for full factor condition

\begin{tabular}{cccccccc}
\hline Operator & $\begin{array}{c}\text { Customer } \\
\text { Orientation } \\
\left(\mathrm{F}_{1}\right)\end{array}$ & $\begin{array}{c}\text { Reduce } \\
\text { Waste } \\
\left(\mathrm{F}_{2}\right)\end{array}$ & $\begin{array}{c}\text { Standard } \\
\text { Product } \\
\left(\mathrm{F}_{3}\right)\end{array}$ & $\begin{array}{c}\text { Pull } \\
\text { System } \\
\left(\mathrm{F}_{4}\right)\end{array}$ & $\begin{array}{c}\text { Task } \\
\text { Management } \\
\left(\mathrm{F}_{5}\right)\end{array}$ & $\begin{array}{c}\text { Efficiency } \\
\text { Score }\end{array}$ & Ranking \\
\hline 1 & 0.82205 & 0.80298 & 0.79634 & 0.55207 & 0.93673 & 0.78204 & 7 \\
2 & 0.70898 & 0.66624 & 0.56942 & 0.98127 & 0.76678 & 0.68031 & 22 \\
3 & 0.64898 & 0.48999 & 0.88049 & 0.53331 & 0.84876 & 0.79237 & 54 \\
4 & 0.82626 & 0.69364 & 0.78316 & 0.59392 & 0.74870 & 0.83238 & 24 \\
5 & 0.56776 & 0.65784 & 0.53428 & 0.67231 & 0.80051 & 0.74238 & 64 \\
6 & 0.81119 & 0.63195 & 0.50240 & 0.46978 & 0.99772 & 0.79576 & 51 \\
7 & 0.48846 & 0.60393 & 0.63427 & 0.44182 & 0.82377 & 0.74894 & 73 \\
8 & 0.88120 & 0.73982 & 0.70552 & 0.77403 & 0.89796 & 0.68031 & 3 \\
9 & 0.65786 & 0.68519 & 0.72085 & 0.85729 & 0.89866 & 0.76397 & 12 \\
10 & 1.00000 & 0.73952 & 0.82644 & 0.77865 & 0.79113 & 0.78267 & 1 \\
11 & 0.83345 & 1.00000 & 0.82307 & 0.52995 & 0.76366 & 0.59845 & 5
\end{tabular}




\begin{tabular}{|c|c|c|c|c|c|c|}
\hline 12 & 0.81329 & 0.65090 & 0.63853 & 0.73542 & 0.62844 & 0.54724 \\
\hline 13 & 0.66039 & 0.75170 & 0.65636 & 0.68757 & 0.82466 & 0.69458 \\
\hline 14 & 0.67479 & 0.66858 & 0.47501 & 0.62355 & 0.64278 & 0.66830 \\
\hline 15 & 0.78009 & 0.73089 & 0.70476 & 0.50087 & 0.47542 & 0.56545 \\
\hline 16 & 0.67064 & 0.74299 & 0.74563 & 0.61535 & 0.73957 & 0.52388 \\
\hline 17 & 0.56485 & 0.85801 & 0.82609 & 0.46934 & 1.00000 & 0.82723 \\
\hline 18 & 0.87582 & 0.63147 & 0.45059 & 0.60798 & 0.93003 & 0.63483 \\
\hline 19 & 0.42949 & 0.80270 & 0.46652 & 0.46931 & 0.78062 & 0.83746 \\
\hline 20 & 0.44328 & 0.82156 & 1.00000 & 0.57071 & 0.69863 & 0.82373 \\
\hline 21 & 0.82209 & 0.71639 & 0.94785 & 0.48704 & 0.86596 & 0.67834 \\
\hline 22 & 0.92350 & 0.83258 & 0.68222 & 0.57020 & 0.92033 & 0.78347 \\
\hline 23 & 0.72160 & 0.84300 & 0.57118 & 0.72008 & 0.69954 & 0.58940 \\
\hline 24 & 0.66403 & 0.54075 & 0.85902 & 0.45195 & 0.90591 & 0.72383 \\
\hline 25 & 0.83473 & 0.40512 & 0.85071 & 0.46625 & 0.85832 & 0.54992 \\
\hline 26 & 0.28842 & 0.43552 & 0.86480 & 0.93963 & 0.74778 & 0.90273 \\
\hline 27 & 0.75293 & 0.69005 & 0.77438 & 0.45571 & 0.85145 & 0.89294 \\
\hline 28 & 0.58735 & 0.60873 & 0.50455 & 0.71057 & 0.86517 & 0.73873 \\
\hline 29 & 0.80466 & 0.59851 & 0.90985 & 0.56664 & 0.79705 & 0.82738 \\
\hline 30 & 0.76296 & 0.76508 & 0.73643 & 1.00000 & 0.60725 & 0.79835 \\
\hline 31 & 0.57417 & 0.69837 & 0.75381 & 0.88642 & 0.86761 & 0.74382 \\
\hline 32 & 0.47364 & 0.78064 & 0.83583 & 0.53479 & 0.78493 & 0.80237 \\
\hline 33 & 0.82461 & 0.67240 & 0.77503 & 0.41254 & 0.86607 & 0.60168 \\
\hline 34 & 0.69773 & 0.66853 & 0.67469 & 0.50049 & 0.92505 & 0.64706 \\
\hline 35 & 0.22824 & 0.80602 & 0.79423 & 0.69832 & 0.73567 & 0.74347 \\
\hline 36 & 0.79014 & 0.63040 & 0.83541 & 0.63965 & 0.97759 & 0.81273 \\
\hline 37 & 0.78685 & 0.57422 & 0.61051 & 0.43637 & 0.95113 & 0.57993 \\
\hline 38 & 0.78330 & 0.64888 & 0.97328 & 0.72668 & 0.86899 & 0.68349 \\
\hline 39 & 0.82205 & 0.76773 & 0.76571 & 0.51264 & 0.84306 & 0.68342 \\
\hline 40 & 0.79738 & 0.68114 & 0.52620 & 0.65225 & 0.74237 & 0.60347 \\
\hline 41 & 0.58896 & 0.42547 & 0.81213 & 0.39350 & 0.82651 & 0.72035 \\
\hline 42 & 0.76864 & 0.50403 & 0.69479 & 0.48779 & 0.64815 & 0.77348 \\
\hline 43 & 0.68182 & 0.69551 & 0.77584 & 0.46894 & 0.74592 & 0.65348 \\
\hline 44 & 0.81119 & 0.68184 & 0.73791 & 0.43364 & 0.89795 & 0.75348 \\
\hline 45 & 0.48846 & 0.62463 & 0.73713 & 0.40080 & 0.74139 & 0.86835 \\
\hline 46 & 0.88120 & 0.71310 & 0.82982 & 0.60984 & 0.77051 & 0.52746 \\
\hline 47 & 0.67428 & 0.61553 & 0.71334 & 0.69937 & 0.70883 & 0.69348 \\
\hline 48 & 0.94385 & 0.66717 & 0.67359 & 0.53139 & 0.82017 & 0.58348 \\
\hline 49 & 0.83345 & 0.95041 & 0.93830 & 0.45424 & 0.79838 & 0.64238 \\
\hline 50 & 0.81329 & 0.66849 & 0.76686 & 0.44194 & 0.72767 & 0.67348 \\
\hline 51 & 0.66039 & 0.73192 & 0.84149 & 0.51568 & 0.97738 & 0.76348 \\
\hline 52 & 0.77014 & 0.73265 & 0.40426 & 0.60852 & 0.61330 & 0.79347 \\
\hline 53 & 0.73359 & 0.71831 & 0.79230 & 0.58891 & 0.48730 & 0.69343 \\
\hline 54 & 0.67064 & 0.76307 & 0.71173 & 0.59777 & 0.78781 & 0.63482 \\
\hline 55 & 0.56485 & 0.91844 & 0.82609 & 0.48739 & 0.95455 & 0.70447 \\
\hline 56 & 0.78040 & 0.83016 & 0.56531 & 0.62253 & 0.99209 & 0.68350 \\
\hline 57 & 0.42949 & 0.71831 & 0.48380 & 0.48736 & 0.79363 & 0.70238 \\
\hline 58 & 0.44328 & 0.77425 & 1.00000 & 0.53418 & 0.78247 & 0.79349 \\
\hline 59 & 0.77435 & 0.68476 & 0.85905 & 0.51637 & 0.95992 & 0.72383 \\
\hline 60 & 0.92350 & 0.71810 & 0.71633 & 0.59213 & 0.77189 & 0.55848 \\
\hline 61 & 0.80892 & 0.74356 & 0.61162 & 0.62471 & 0.66261 & 0.52791 \\
\hline 62 & 0.66403 & 0.55877 & 0.77480 & 0.42704 & 0.85280 & 0.75708 \\
\hline 63 & 0.83473 & 0.52260 & 0.73619 & 0.44960 & 0.87393 & 0.52855 \\
\hline 64 & 0.35995 & 0.45891 & 0.72502 & 0.67886 & 0.79569 & 0.76348 \\
\hline 65 & 0.75293 & 0.71694 & 0.69694 & 0.59441 & 0.85145 & 0.79835 \\
\hline 66 & 0.58735 & 0.66407 & 0.53462 & 0.71057 & 0.93047 & 0.75708 \\
\hline 67 & 0.77241 & 0.58805 & 0.68221 & 0.65387 & 0.81592 & 0.67835 \\
\hline 68 & 0.76296 & 0.76508 & 0.80046 & 0.75862 & 0.76919 & 0.78744 \\
\hline 69 & 0.57417 & 0.65181 & 0.75381 & 0.69302 & 0.76639 & 0.65053 \\
\hline 70 & 0.47364 & 0.66640 & 0.86369 & 0.55262 & 0.79894 & 0.75708 \\
\hline 71 & 0.73729 & 0.70766 & 0.70034 & 0.47206 & 0.88096 & 0.61749 \\
\hline
\end{tabular}




\begin{tabular}{llllllll}
72 & 0.69773 & 0.69678 & 0.68433 & 0.55053 & 0.79290 & 0.65083 & 45 \\
73 & 0.35188 & 0.79710 & 0.77609 & 0.38854 & 0.76713 & 0.75708 & 69 \\
74 & 0.79014 & 0.69435 & 0.68071 & 0.60251 & 0.65071 & 0.69824 & 47 \\
75 & 0.78685 & 0.63560 & 0.75511 & 0.45383 & 0.95113 & 0.64963 & 27 \\
\hline
\end{tabular}

